\title{
GEOHYDROLOGIC DATA FROM PORT ROYAL SOUND, BEAUFORT COUNTY, SOUTH CAROLINA
}

U.S. GEOLOGICAL SURVEY

Open-File Report 86-497

Prepared in cooperation with 
This report uses inch-pound units as the primary system of measurements. Metric units are used for water chemistry measurements. To convert inch-pound units to equivalent metric units, mulitply by the following factors:

$\begin{array}{lc}\text { Multiply } & \text { By } \\ \text { Foot (ft) } & 0.3048 \\ \text { Inch (in.) } & 25.4\end{array}$

Multiply Inch (in.)
To Obtain

meter $(m)$

millimeter $(\mathrm{mm})$

Temperature in degrees Celsius $\left({ }^{\circ} \mathrm{C}\right)$ can be converted to degrees Fahrenheit $\left({ }^{0} \mathrm{~F}\right)$ as follows:

$$
{ }^{0} \mathrm{C}=0.556\left({ }^{\circ} \mathrm{F}-32\right)
$$

Sea level: In this report "sea level" refers to the National Geodetic vertical Datum of 1929 (NGVD of 1929)--a geodetic datum derived from a general adjustment of the first-order level nets of both the United States and Canada, formerly called "Mean Sea Level of 1929." 


\title{
GEOHYDROLOGIC DATA FROM PORT ROYAL SOUND, BEAUFORT COUNTY SOUTH CAROLINA
}

By Ronald A. Burt, Donna L. Belval, Michael Crouch, and W. Brian Hughes

\author{
ABSTRACT
}

Nine offshore wells were drilled through overlying sediments into the Upper Floridan aquifer in Port Royal Sound, South Carolina and the adjacent Atlantic Ocean, to obtain geologic, hydrologic, and water-quality data.

The Upper Floridan aquifer consists predominantly of light-gray, poorly consolidated, fossiliferous limestone. In the Port Royal Sound area, the Upper Floridan is overlain by olive-gray, medium to coarse sand and silty sand. Falling-head permeameter tests on these ${ }_{4}$ overlying clastic sediments indicate hydraulic conductivities of $4.2 \times 10^{-4}$ to 3.1 feet per day. Other geologic and hydrologic data, including geophysical logs, sieve analyses, and detailed core descriptions were obtained, along with continuous water level records of the wells, tidal records, and barometric pressure records.

Water collected from the Upper Floridan aquifer beneath Port Royal Sound and the ocean ranged in concentration of chloride from 54 to 12,000 milligrams per liter. Measured pH ranged from 6.8 to 8.4 , and alkalinity ranged from 122 to 368 milligrams per liter as $\mathrm{CaCO}_{3}$. Other water quality data obtained include temperature, specific conductance, carbon-13, carbon-14, tritium, deuterium, oxygen-18, dissolved oxygen, dissolved solids, nitrogen species, phosphorus, organic carbon, cyanide, sulfide, calcium, magnesium, sodium, potassium, sulfate, fluoride, silica, bromide, iodide, and selected trace metals.

\section{INTRODUCTION}

An initial collection of geologic, hydrologic, and water quality data has been compiled for the Beaufort-Jasper Counties Regional Ground Water Study, a cooperative project funded by the South Carolina Water Resources Commission and the U.S. Geological Survey. The study was designed to describe the water quality and hydrogeologic characteristics of the Upper Floridan aquifer and its overlying confining bed in Beaufort and Jasper Counties, South Carolina, with particular emphasis on the saltwater-freshwater zone of mixing in the vicinity of Port Royal Sound.

This report is restricted to information obtained during the drilling of nine offshore wells in Port Royal Sound and the adjacent Atlantic Ocean. The data were collected between July 16 and October 5, 1984. All reported times refer to Eastern Standard Time. This report will be supplemented by subsequent publications presenting data and interpretations from onshore wells near Port Royal Sound. 
The study area is located in the coastal region of southern South Carolina (fig. l) and consists of barrier islands and salt marshlands surrounding Port Royal Sound, the Broad River, the Beaufort River, and several creeks. The area includes the resort community of Hilton Head Island, the towns of Beaufort and Port Royal, and the Parris Island Marine Corps Depot, as well as a number of less populated areas.

The Beaufort-Jasper Counties Regional Ground Water Study was initiated because of concern for the future of the ground-water resources of the Upper Floridan aquifer. This high-yield aquifer currently supplies nearly all the potable water used by the resort community of Hilton Head Island. Previous studies showed that saline water stored in the Upper Floridan aquifer beneath Port Royal Sound may move foward the ground-water production areas of Hilton Head Island (Hayes, 1979; Counts and Donsky, 1963).

Several prior studies pertained to the ground-water resources and geology of the study area. Published reports were by Back and others (1970), Callahan (1964), Colquhoun and others (1969), Comer (1973), Cooke (1936), Cooke and MacNeil (1952), Counts and Donsky (1963), Counts (1958), Duncan (1972), Hayes (1979), Hazen and Sawyer (1956), Hazen and Sawyer (1957), McCollum and Counts (1964), Mundorff (1944), Nuzman (1970 and 1972), Siple (1956), Siple (1960), Siple (1967), Stringfield (1966), and Warren (1944). (The tables follow the text and references).

\section{DRILLING AND DATA COLLECTION}

Nine wells were drilled offshore in Port Royal Sound and the adjacent Atlantic Ocean (fig. 2) by the U.S. Army Corps of Engineers "Seahorse" drilling ship. The Seahorse is a self-propelled, jack-up rotary drilling rig that is supported above the water surface during drilling operations by three legs.

Test well locations were surveyed with a Miniranger III Automated Positioning System (Motorola*) or with a theodolite to obtain latitude and longitude. Altitudes of test wells and tide gages were surveyed to an accuracy \pm 0.16 foot by the South Carolina Geodetic Survey. Test well altitudes and locations are given in table 1 , along with well construction data.

Test wells are identified by county labels, according to an established convention in South Carolina, which consist of a three-letter prefix followed by a number. The prefix is an abbreviation of the name of the county in which a well is located. All offshore test wells referred to in this report are labeled as Beaufort County wells with the prefix BFT followed by numbers 1672 through 1680 assigned consecutively to the wells in the order in which they were drilled.

*The use of the brand names, Motorola, Leopold and Stevens, Soiltest and Orion, in this report is for the purpose of identification only and does not constitute endorsement by the U.S. Geological Survey or the South Carolina Water Resources Commission. 


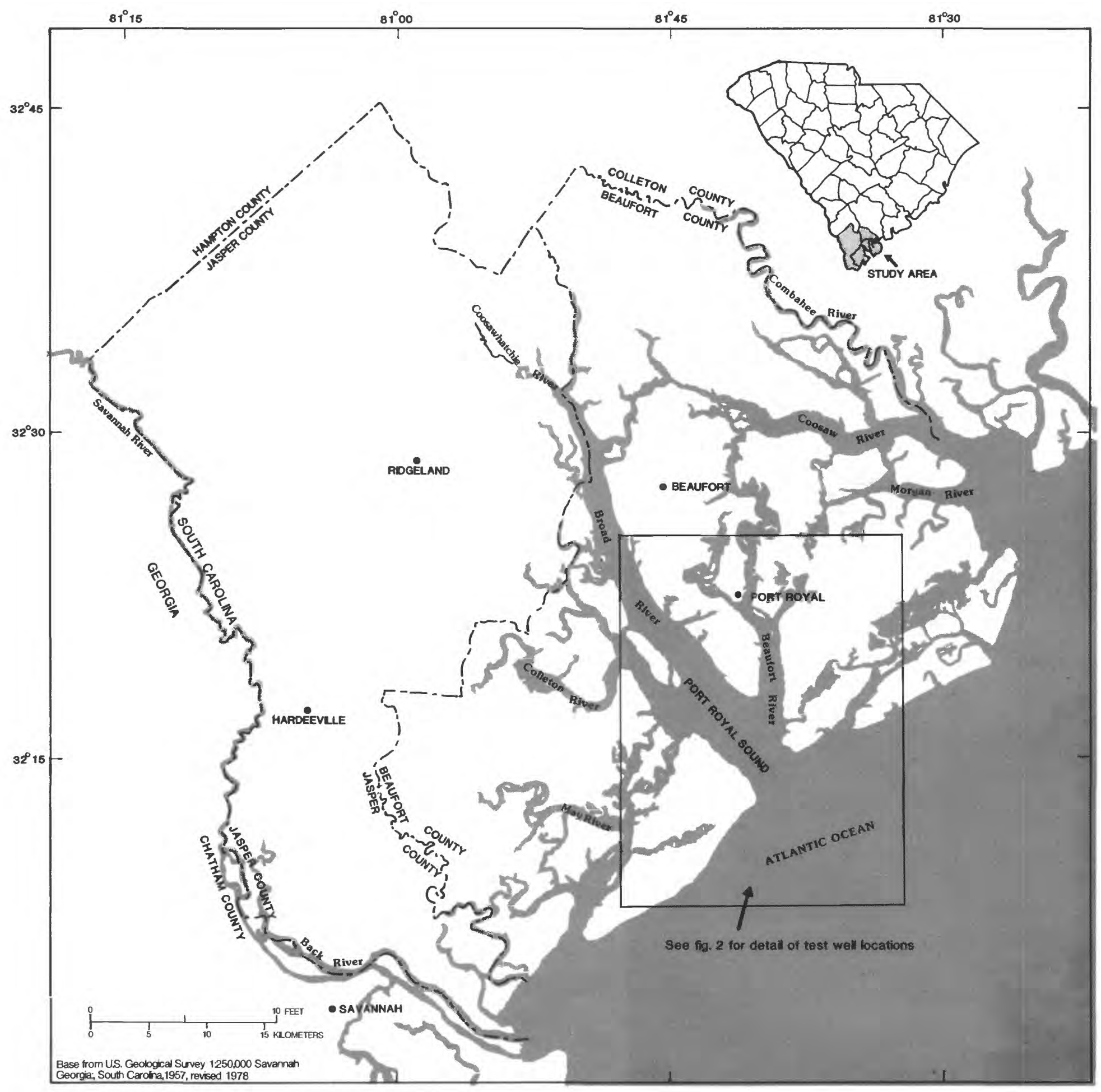

Figure 1.--Beaufort-Jasper Counties Regional Ground Water Study area. 


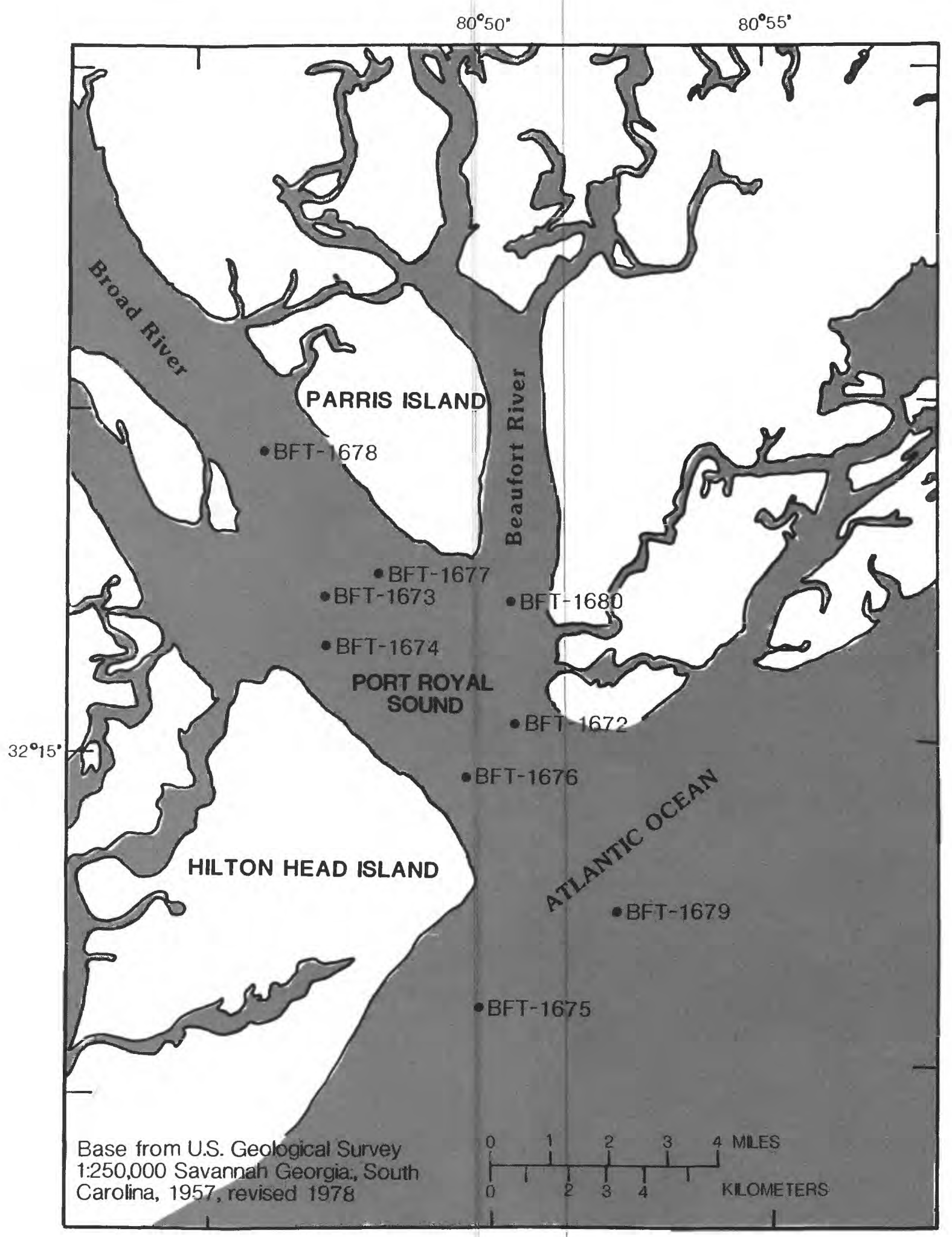

Figure 2.--Locations of Port Royal Sound test wells. 
Each well was cased and grouted through the overlying confining bed and a few feet into the Upper Floridan aquifer to restrict the well to that aquifer prior to deeper drilling. The portion of each well in the Upper Floridan was of open-hole construction. At the conclusion of data collection, each well was filled from its bottom to the lower end of the casing with sand and gravel, and then plugged with grout. The casing was then cut off at the floor of the Sound. Well construction diagrams are presented in plates $1-9$.

Geologic and water samples were collected and hydrologic measurements made during the drilling of the wells. Cores and drill cuttings were retrieved while drilling, and geophysical logs were obtained at the completion of each well. Water level fluctuations in the wells and tidal fluctuations in Port Royal Sound were simultaneously recorded. Water samples for chemical analysis were collected at various depths during and after the drilling of each well. The details and results of these procedures follow.

\section{$\underline{\text { Geologic Data }}$}

Geologic data were obtained from sediment and rock samples collected during the well drilling process and by geophysical logging of the boreholes.

Sediment and rock samples were obtained by three methods:

(1) conventional-rotary coring,

(2) split-spoon sampling, and

(3) collection of drill cuttings as they emerged at the wellhead.

A total of 410 feet of conventional core was obtained with a 10-foot, $4 \frac{1}{2}-$ inch diameter core barrel. Approximately 10 additional feet of core were recovered from BFT-1672 and BFT-1673 by driving a 7-foot long, 4-inch diameter split-spoon sampler into the formation. All cores were sealed in plastic to retard drying. Where cores were not taken, a sieve was used to catch cuttings at the wellhead during drilling.

Sediment and rock samples were described while still wet, using a binocular microscope. Rock names used in the lithologic descriptions in table 2 are after Travis (1955). Textures were estimated by using Wentworth's grade scale (1922). Colors and color identification codes are from the Rock-Color Chart (1980). Twenty-two sections of core were sieved to determine the grain-size distributions. U.S. standard sieves No. 40 $(0.42 \mathrm{~mm})$, No. $60(0.25 \mathrm{~mm})$, No. $100(0.149 \mathrm{~mm})$, No. $200(0.074 \mathrm{~mm})$, and a pan were used to separate the different size grades. The sieve analyses were made in the Civil Engineering Department laboratory at the University of South Carolina. The results of these analyses are given in Table 3 and graphically illustrated in figure 3-10.

Geophysical logs were made of each of the wells. Gamma-ray, spontaneous-potential, resistance, fluid resistivity, caliper, and temperature logs are presented in plates 1-9. Although several fluid-resistivity logs were made on some wells, only the last one made after completion of each well is shown. 


\section{Hydrologic Data}

Hydrologic data were collected at all of the offshore wells. Water levels were measured at 6-minute intervals over the duration of at least one complete tidal cycle in each of the wells using Leupold and Stevens Analog to Digital Recorders. Tidal fluctuations of water level in the Sound or Ocean were measured simultaneously with well water level measurements using a recorder installed on a gaging station attached to the Seahorse. A permanent tide level station located on the Beaufort River near the city of Beaufort was also monitored. Continuously recorded water level data from wells and tide gages are presented in hydrograph form in figures $11-19$.

Barometric-pressure measurements, corresponding to the times of water level records, were obtained from the National Weather Service at the U. S. Marine Corps Air Station in Beaufort. These are presented in table 4.

Permeameter tests were made on selected cores. The tests were of the falling head type (ASTM D2434-65T), using Soiltest Model K-605 permeameters. The effective porosities of these cores were determined gravimetrically. The results of the permeameter tests and porosity determinations are shown in table 5.

\section{Water Quality Data}

Water quality measurements were made and water samples for chemical and isotopic analyses were collected at each of the wells. The samples were collected from discrete depth intervals in the aquifer by isolating the interval of interest in the well and withdrawing water from that interval with a submersible pump. Conductivity, $\mathrm{pH}$, temperature, and concentration of dissolved oxygen in the sample stream were monitored at the wellhead until they became constant, indicating that the retrieved sample was representative of water from the aquifer in the isolated interval. With the exception of BFT-1679, two samples were collected in this manner from each of the wells; one sample was collected from an interval between 10 and 20 feet below the top of the Upper Floridan aquifer, and the other was collected from an interval between 50 and 100 feet below the top of the Upper Floridan aquifer. Only the upper interval was sampled in BFT-1679. The results of all measurements, chemical analyses, and isotope analyses are presented in table 6.

During drilling through the limestone of the Upper Floridan aquifer, the natural flow of ground water from the aquifer into the borehole was used to flush drill cuttings from the well. The ground water, along with the drill cuttings, was air-lifted from the bottom of the hole, up through the annulus of the well, and discharged at the wellhead. Samples of this discharge water were collected at 5-foot or 10-foot depth intervals for analysis of chloride concentration. These analyses are presented in table 7. 
Several hours after the completion of drilling each well, water samples from the wellbore were collected with a Kemmerer-type point sampler. These samples were obtained from several depths determined by evaluation of down-hole fluid-resistivity logs. The fluid-resistivity logs record variations in water quality along the wellbore. Sample depths were chosen to effectively describe these variations. The wellbore samples were analyzed for concentration of chloride. The results of these analyses are presented in table 8.

Specific conductance was measured in situ in the wellbores of BFT-1678 and BFT-1679. These data are presented in table 9.

Inorganic and organic constituent analyses were performed by the laboratory of the U.S. Geological Survey in Doraville, Ga. and by the laboratory of the South Carolina Water Resources Commission in Columbia, S.C. The U.S. Geological Survey laboratory measured some constituents not measured by the South Carolina Water Resources Commission laboratory; these included nitrate, nitrite, ammonia, total dissolved phosphate, orthophosphate, dissolved organic carbon, and most of the trace constituents.

Samples were processed, preserved, and analyzed for chemical constituents by established procedures (Skougstad and others, 1979). Concentrations of sulfide were determined by the South Carolina Water Resources Commission laboratory, using a sulfide-ion electrode, (ORION Research, Inc., Model 94-16). Values for pH, temperature, specific conductance, dissolved oxygen, alkalinity, bicarbonate and carbonate were obtained in the field (Wood, 1976). Alkalinity analyses by the U.S. Geological Survey followed an incremental titration method (Barnes, 1964), while those done by the South Carolina Water Resources Commission followed a fixed-endpoint ( $\mathrm{pH}$ 4.5) titration method.

Samples for deuterium, oxygen-18, tritium, carbon-13, and carbon-14 were collected and processed according to established procedures (Busby and others, 1983). These isotope analyses were made by Isotope Lab in Waterloo, Ontario, Canada. Selected carbon-14 and carbon-13 analyses were repeated by Tritium Laboratory in Miami, Florida using an alternative method of carbon extraction. Samples analyzed for carbon-14 and carbon-13 by Isotope Lab were processed in the field by precipitating the inorganic carbon species as strontium carbonate directly from the water sample (Busby and others, 1983). The strontium carbonate was prepared and analyzed for carbon-14 and carbon-13 at the laboratory. In contrast, unprocessed water samples were provided to Tritium Laboratory where a gas-stripping technique was employed for extraction of the inorganic carbon species prior to analysis (Miami Radiocarbon/Tritium Laboratory, 1973). A comparison of these results is presented in table 10.

The analytical values for the chemical constituents are reported as milligrams per liter ( $\mathrm{mg} / \mathrm{L}$ ) or micrograms per liter ( $\mathrm{ug} / \mathrm{L}$ ) except as noted here. Specific conductance is reported as microsiemens per $\mathrm{cm}$ at $25^{\circ} \mathrm{C}$. In most cases the sample $\mathrm{pH}$ was measured to $\pm 0.02 \mathrm{pH}$ units and, therefore, is reported to two decimal places. Carbon-13, deuterium, and oxygen-18 are 
reported as a $\delta$-value and expressed in parts per thousand (per mil) relative to an internationally accepted standard. These values are calculated from the equation,

$$
\delta_{x}=\frac{R_{x}}{R_{\text {std }}}-1000 \quad \text { (Fritz and Fontes, 1980), }
$$

where $R_{X}$ is the sample isotopic ratio $\left({ }^{13} \mathrm{C} /{ }^{12} \mathrm{C},{ }^{2} \mathrm{H} /{ }^{1} \mathrm{H},{ }^{18} \mathrm{O} /{ }^{16} \mathrm{O}\right)$

Rstd (Deuterium, Oxygen-18) is the Vienna Standard Mean Ocean Water ( $V$-SMOW) standard

Rstd (Carbon-13) is the Pee Dee Belemnite (PDB) standard

Carbon-14 is expressed as the percentage of modern atmospheric carbon-14 based on the National Bureau of Standards oxalic acid standard. Tritium is reported in terms of Tritium Units (T.U.). One T.U. corresponds to one in $10^{18}$ hydrogen atoms. 
Miami Radiocarbon/Tritium Laboratory, 1973, Miami Radiocarbon/Tritium Laboratory $\mathrm{CO}_{2}$ Extraction System Manual.

Back, William, Hanshaw, B.B., and Rubin, M., 1970, Carbon-14 ages related to occurrence of salt water: American Society of Civil Engineers Proceedings, Journal of the Hydraulics Division, No. 1970.

Barnes, Ivan, 1964, Field measurement of alkalinity and pH: U.S. Geological Survey Water-Supply Paper 1535-H, p. 17.

Busby, J. F., Lee, R. W., and Hanshaw, B. B., 1983, Major geochemical processes related to the hydrology of the Madison Aquifer System and associated rocks in parts of Montana, South Dakota, and Wyoming: U.S. Geological Survey Open-File Report 83-4093.

Callahan, J.T., 1964, The yield of sedimentary aquifers of the coastal plain southeast river basins: U.S. Geological Survey Water-Supply Paper 1669-W, $56 \mathrm{p}$.

Colquhoun, D.J., Heron, S.D., Jr., Johnson, H.S., Jr., Pooser, W.K., and Siple, G.E., 1969, Up-dip Paleocene-Eocene stratigraphy of South Carolina reviewed: Columbia, S.C., South Carolina Geological Survey, Geologic Notes, v. 13, no. 1, p. 1-25.

Comer, C.D., 1973, Upper Tertiary stratigraphy of the lower coastal plain of South Carolina: University South Carolina, Department of Geology, M.S. Thesis, $19 \mathrm{p}$.

Cooke, C.W., 1936, Geology of the coastal plain of South Carolina: U.S. Geological Survey Bulletin 867, $196 \mathrm{p}$.

Cooke, C.W., and MacNeil, F.S., 1952, Tertiary stratigraphy of South Carolina: U.S. Geological Survey Professional Paper 243-B, p. 19-29.

Counts, H.B., 1958, The quality of ground water in the Hilton Head Island area, Beaufort County, South Carolina: Atlanta, Ga., Georgia Department of Mines, Mining and Geology, Georgia Mineral Newsletter, v. 11 , no. 2, p. 50-51.

Counts, H.B., and Donsky, Ellis, 1963, Salt-Water encroachment geology and ground-water resources of Savannah Area, Georgia and South Carolina: U.S. Geological Survey Water-Supply Paper 1611.

Duncan, D.A., 1972, High resolution seismic study, in Port Royal Sound Environmental Study: South Carolina Water Resources Commission, p. 85-106.

Fritz, P., and Fontes, J.C., 1980, Handbook of environmental isotope geochemistry: Amsterdam, The Netherlands, Elsevier Scientific Publishing Company, 545 p.

Hayes, L.R., 1979, The ground-water resources of Beaufort, Colleton, Hampton and Jasper Counties, South Carolina: South Carolina Water Resources Commission Report Number 9.

Hazen, Richard, and Sawyer, A.W., 1956, Water supply in the vicinity of Beaufort, South Carolina: Engineering report to the Bureau of Yards and Docks, Department of the Navy, contract NBY-4440, 40 p.

-.-- 1957, Supplementary report on water supply in the vicinity of Beaufort, South Carolina: Engineering report to the Bureau of Yards and Docks, Department of the Navy, contract NBY-4440, 32 p.

McCollum, M.J., Counts, H.B., 1964, Relations of saltwater encroachment to the major aquifer zones, Savannah area, Georgia and South Carolina: U.S. Geological Survey Water-Supply Paper 1613-D, 26 p. 
REFERENCES--Continued

Mundorff, M.J., 1944, Ground water in the Beaufort area, South Carolina: U.S. Geological Survey, Report to the U.S. Navy Department.

Nuzman, C.E., 1970, BASF Corporation aquifer test Port Victoria, South Carolina: Kansas City, Mo., Layne-Western Company, Inc., Engineering report, $71 \mathrm{p}$.

---- 1972, Water-supply study Hilton Head Isalnd, South Carolina: Kansas City, Mo., Layne-Western Company, Inc., Engineering Report, 40 p.

Rock-Color Chart Committee, 1980, Rock-color chart: Netherlands, HuyskesEnschede.

Siple, George E., 1956, Memorandum on the geology and ground-water resources of the Parris Island area, South Carolina: U.S. Geological Survey Open-File Report, $29 \mathrm{p}$.

---- 1960, Geology and ground-water conditions in the Beaufort area, South Carolina: U.S. Geological Survey Open-File Report, $124 \mathrm{p}$.

-.-- 1967, Salt-water encroachment in coastal South Carolina: Columbia, S.C., South Carolina Geological Survey, Geologic Notes, v. 11, no. 2, p. $21-36$.

Skougstand, M.W., Fishman, M.J., Friedman, L.C., Erdmann, D.E., and Duncan, S.S., 1979, Methods for the analyses of inorganic substances in water and fluvial sediment: Techniques of Water-Resources Investigations of the U.S. Geological Survey, book 5, chap A, $626 \mathrm{p}$. Stringfield, V.T., 1966, Artesian water in Tertiary limestone in the southeastern states: U.S. Geological Survey Professional Paper 517, 226 p.

Travis, R.B., 1955, Classification of rocks: Quarterly of the Colorado School of Mines, v. 50, no. 1, $98 \mathrm{p}$.

Warren, M.S., 1944, Artesian water in southeastern Georgia, with special reference to the coastal area: Atlanta, Ga., Georgia Geological Survey Bulletin 49, $40 \mathrm{p}$.

Wentworth, C.K., 1922, A scale of grade and class terms for clastic sediments: Journal of Geology, v. 30, p. 377-392.

White, W.B., 1969 , Conceptual models for carbonate aquifers: Ground Water, v. 7, no. 3.

Wood, W.W., 1976, Guidelines for collection and field analysis of groundwater samples for selected unstable constituents: U.S. Geological Survey Techniques of Water-Resources Investigations, book 1, chap. D2, $24 \mathrm{p}$.

Woollard, G.P., Bonini, W.E., and Meyer, R.P., 1957, A seismic refraction study of the subsurface geology of the Atlantic Coastal Plain and Continental Shelf between Virginia and Florida: Madison, Wis., University of Wisconsin, Department of Geology, $128 \mathrm{p}$. 


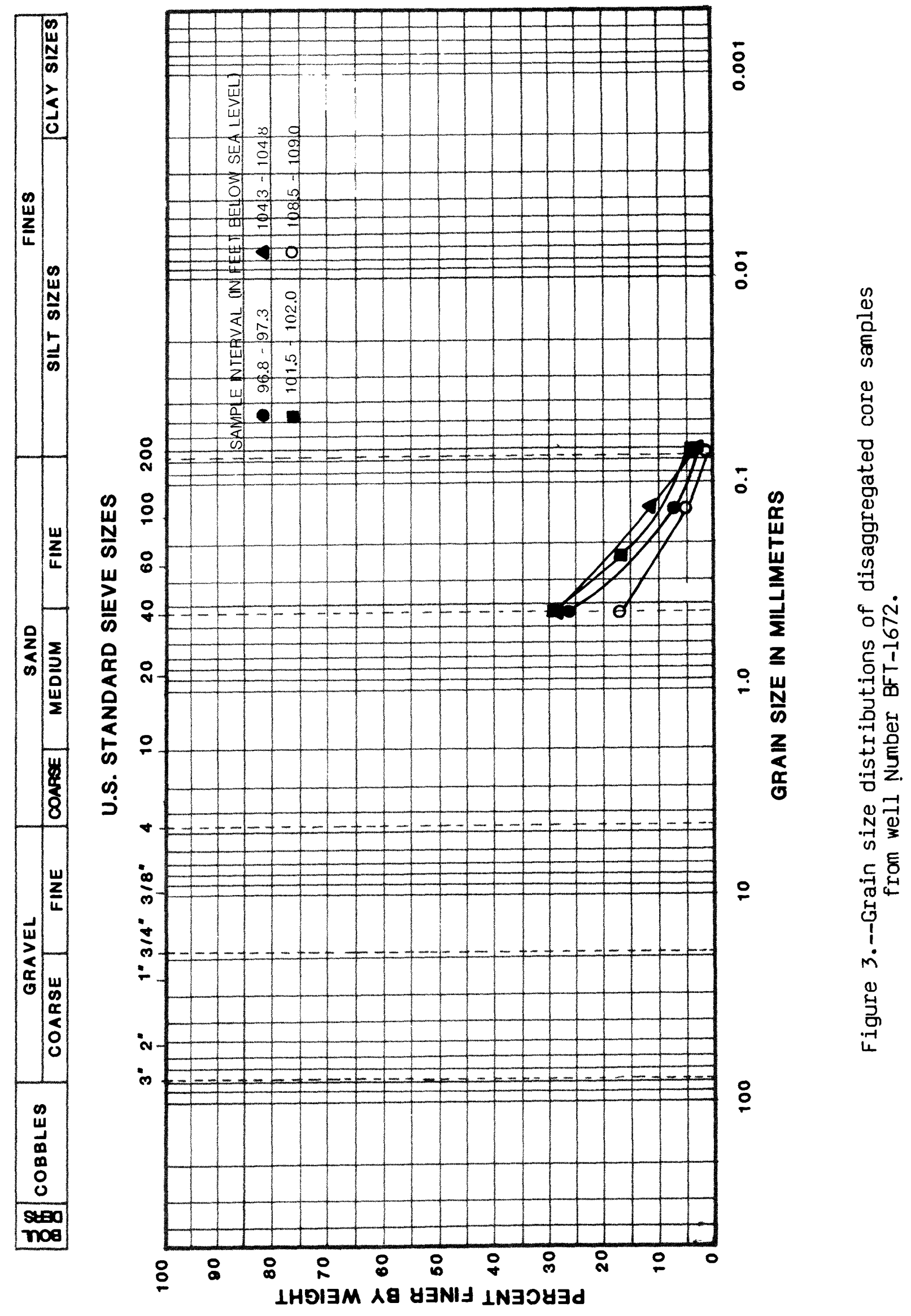




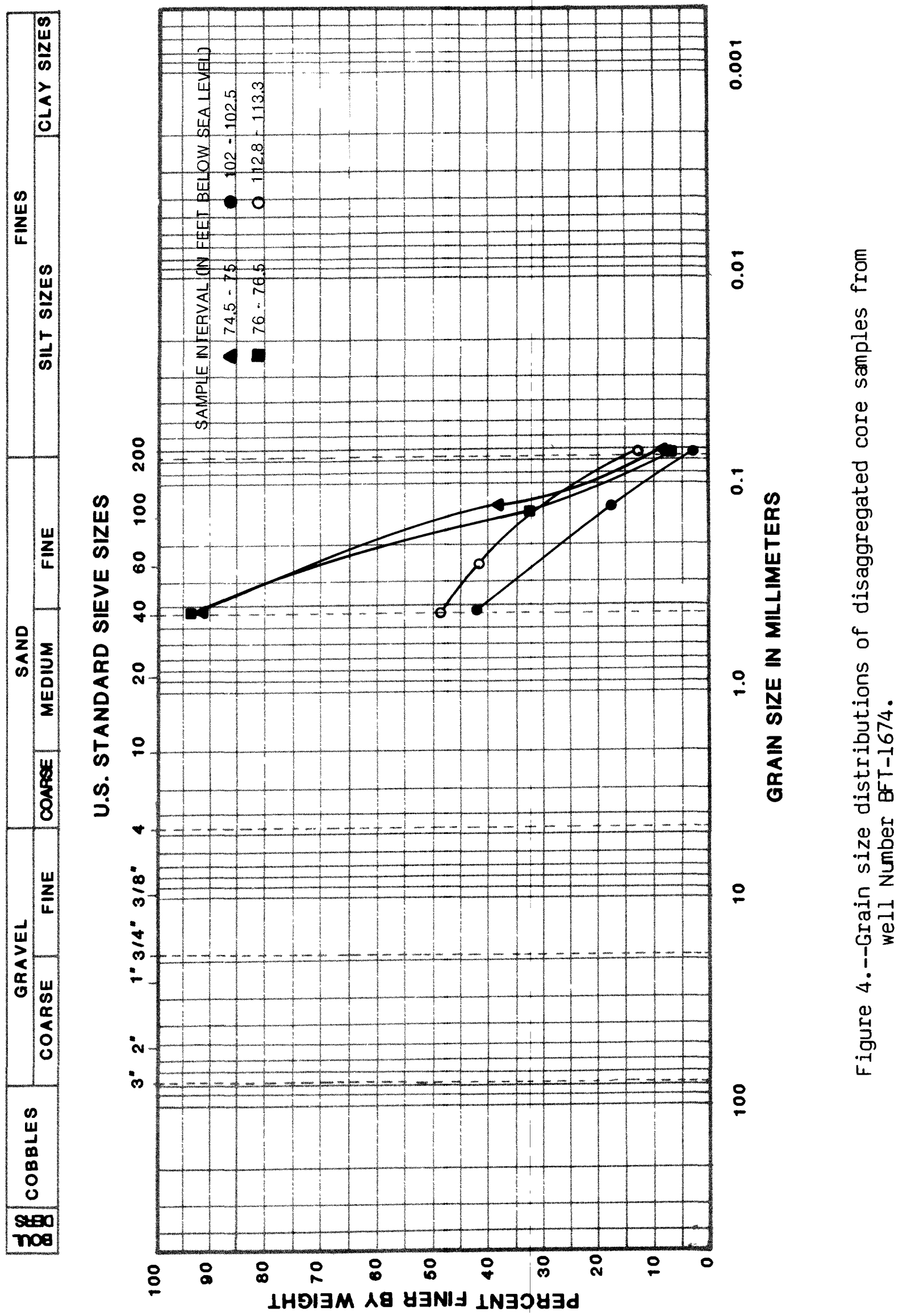




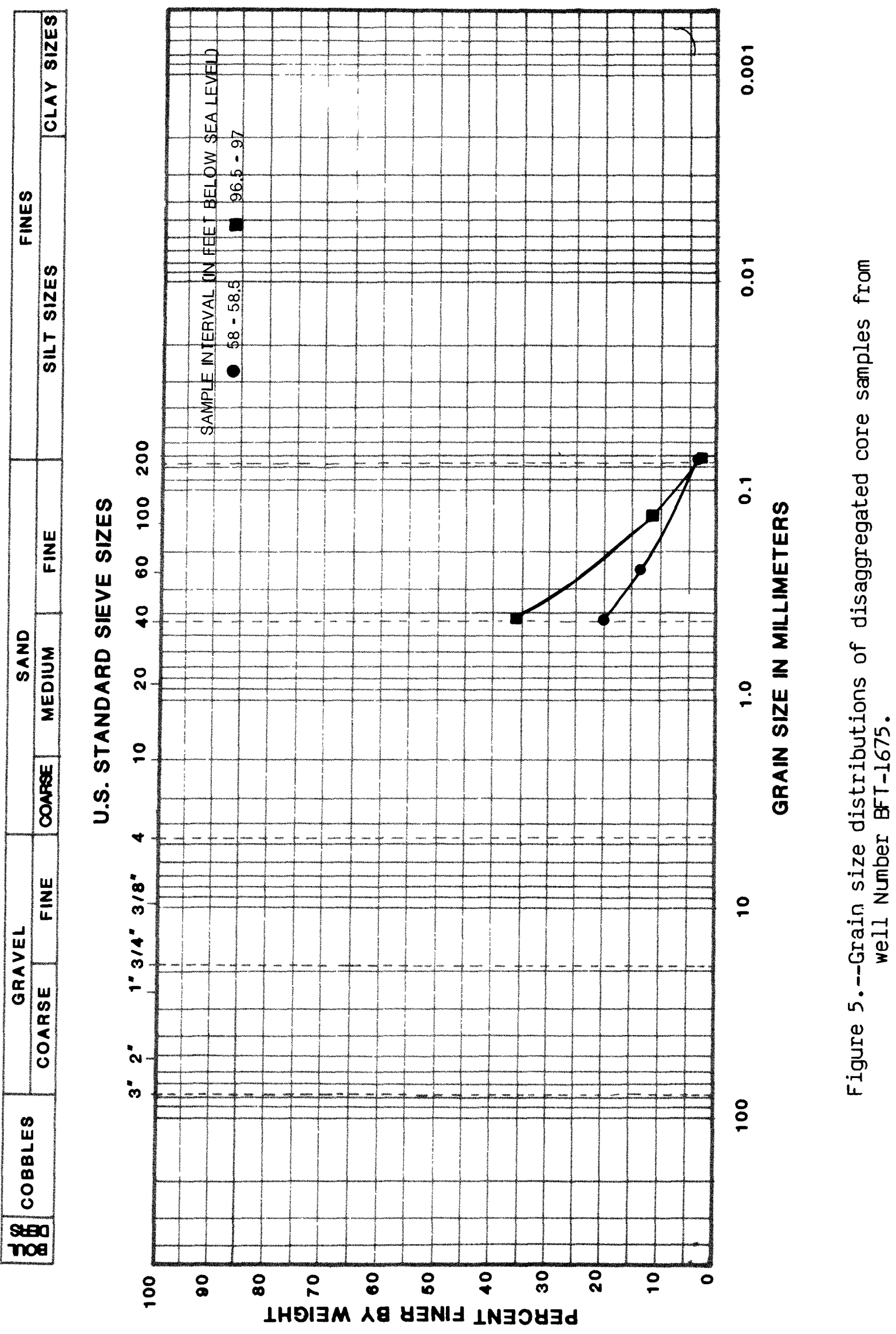




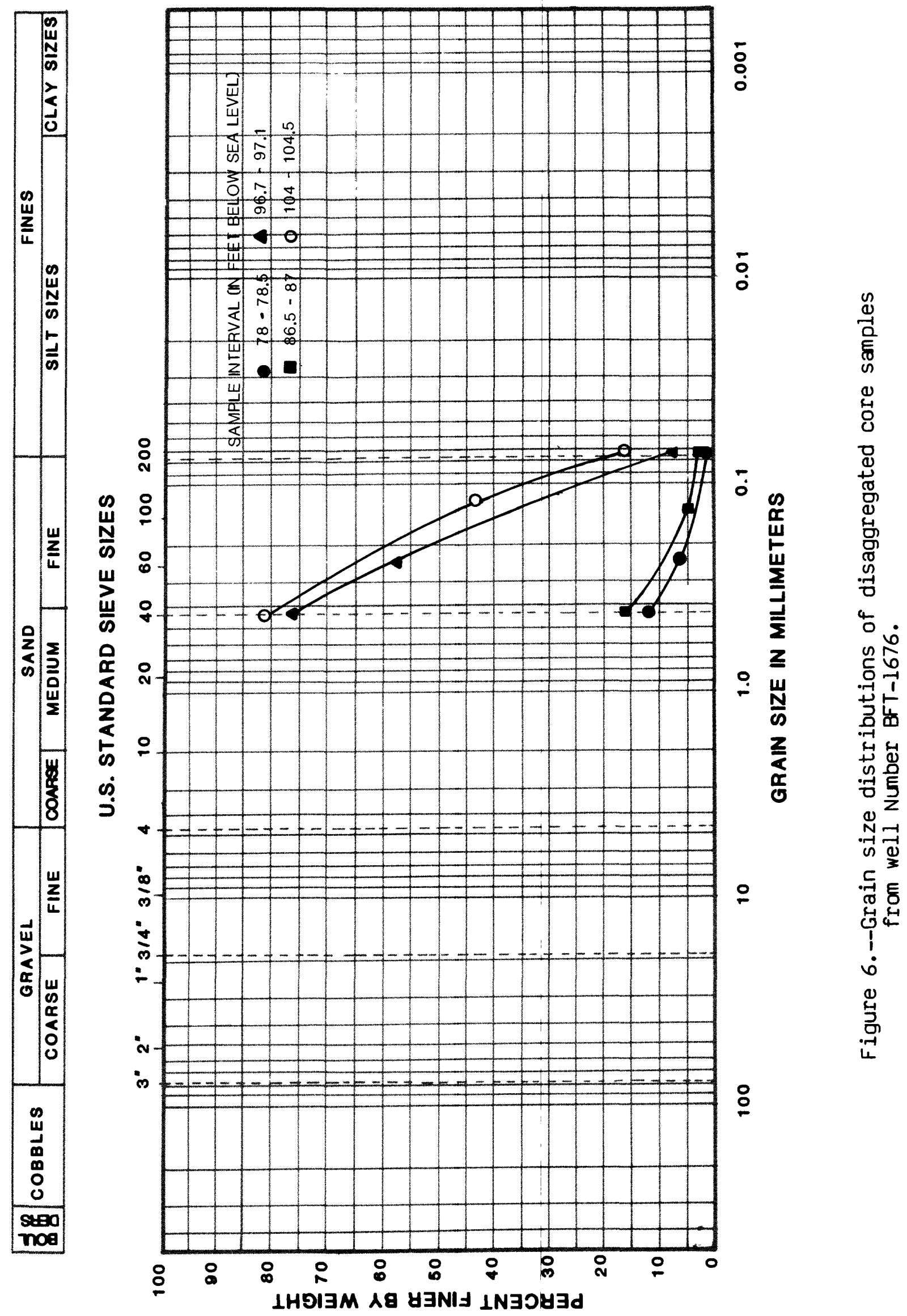




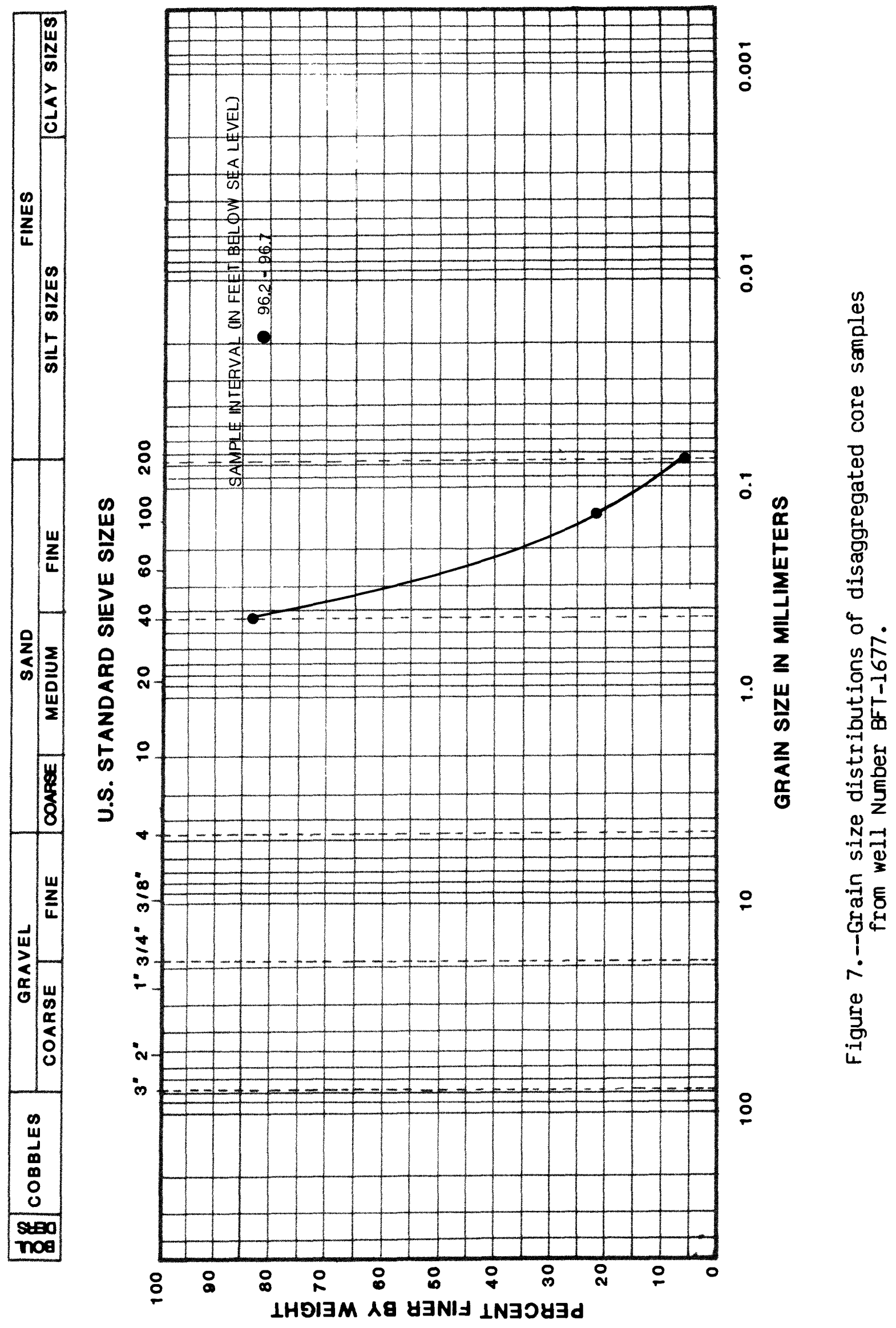




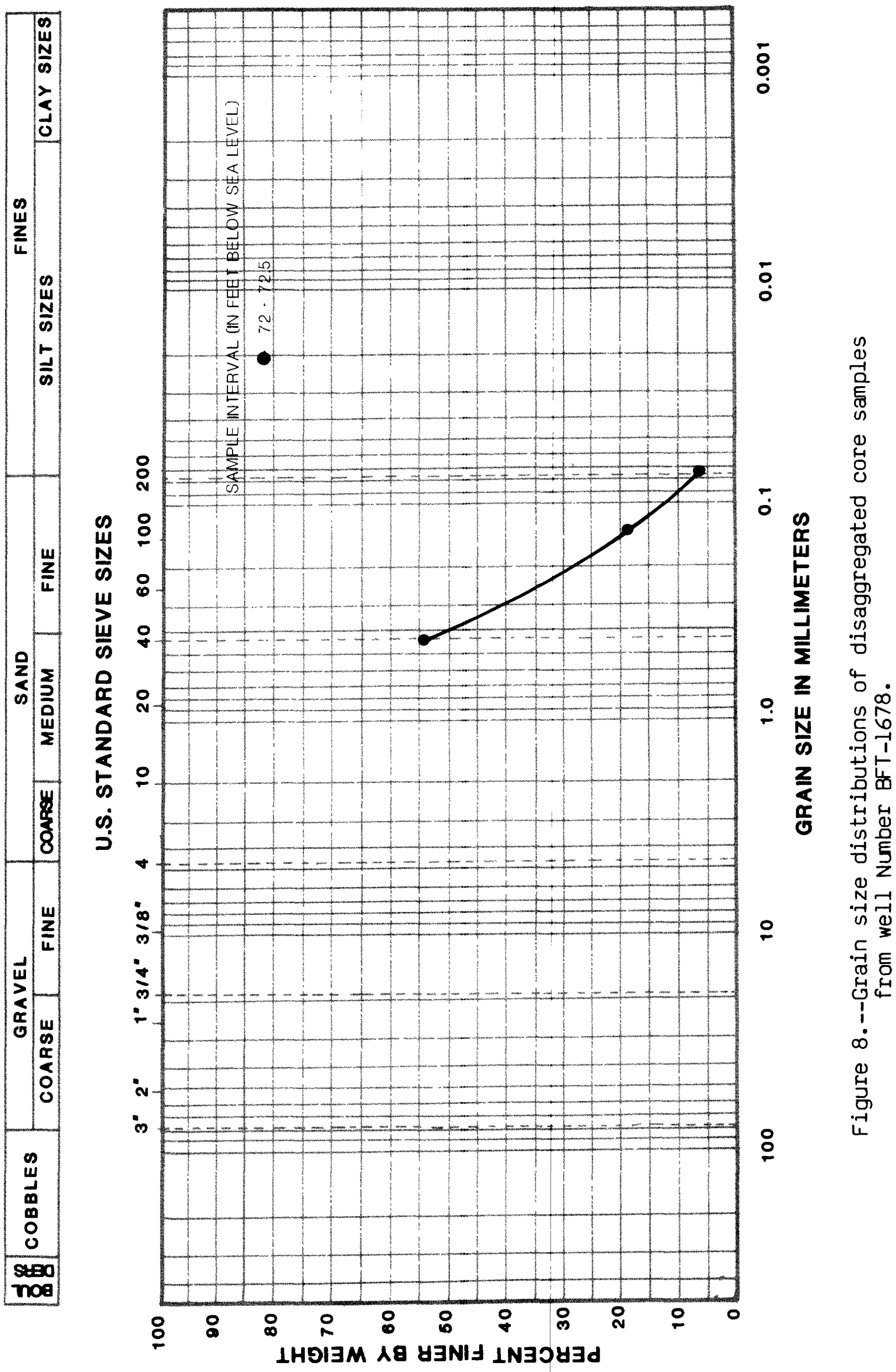




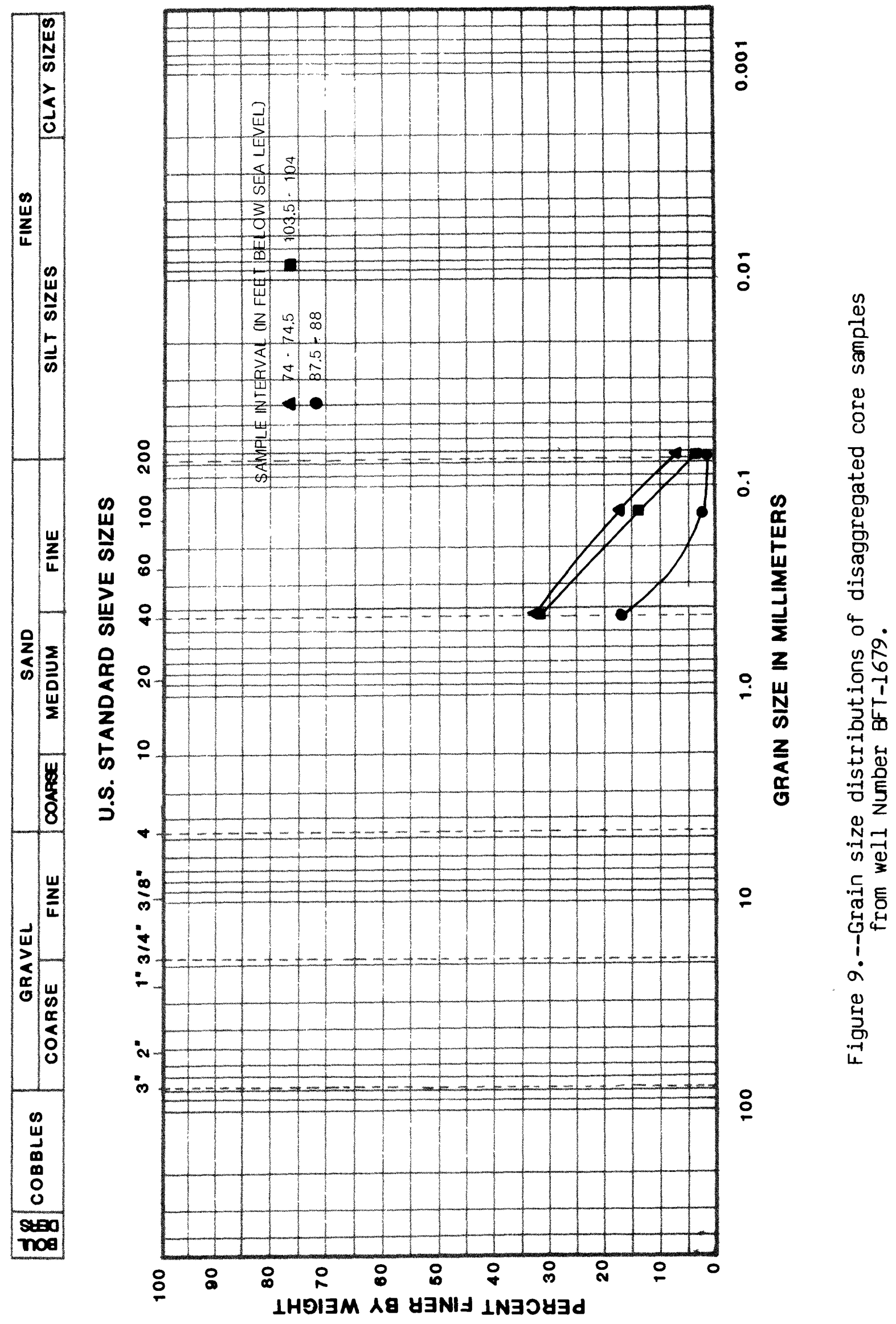




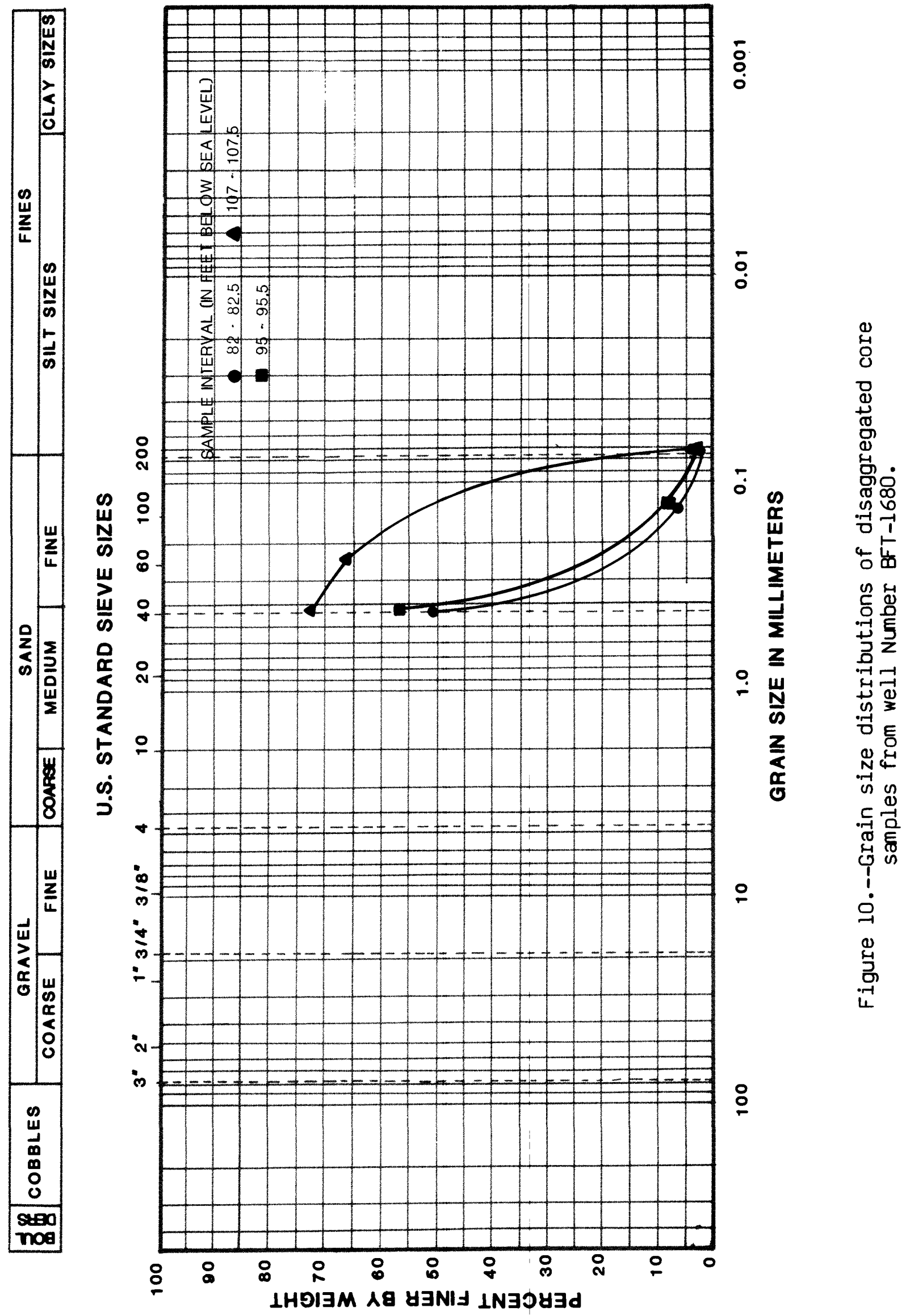




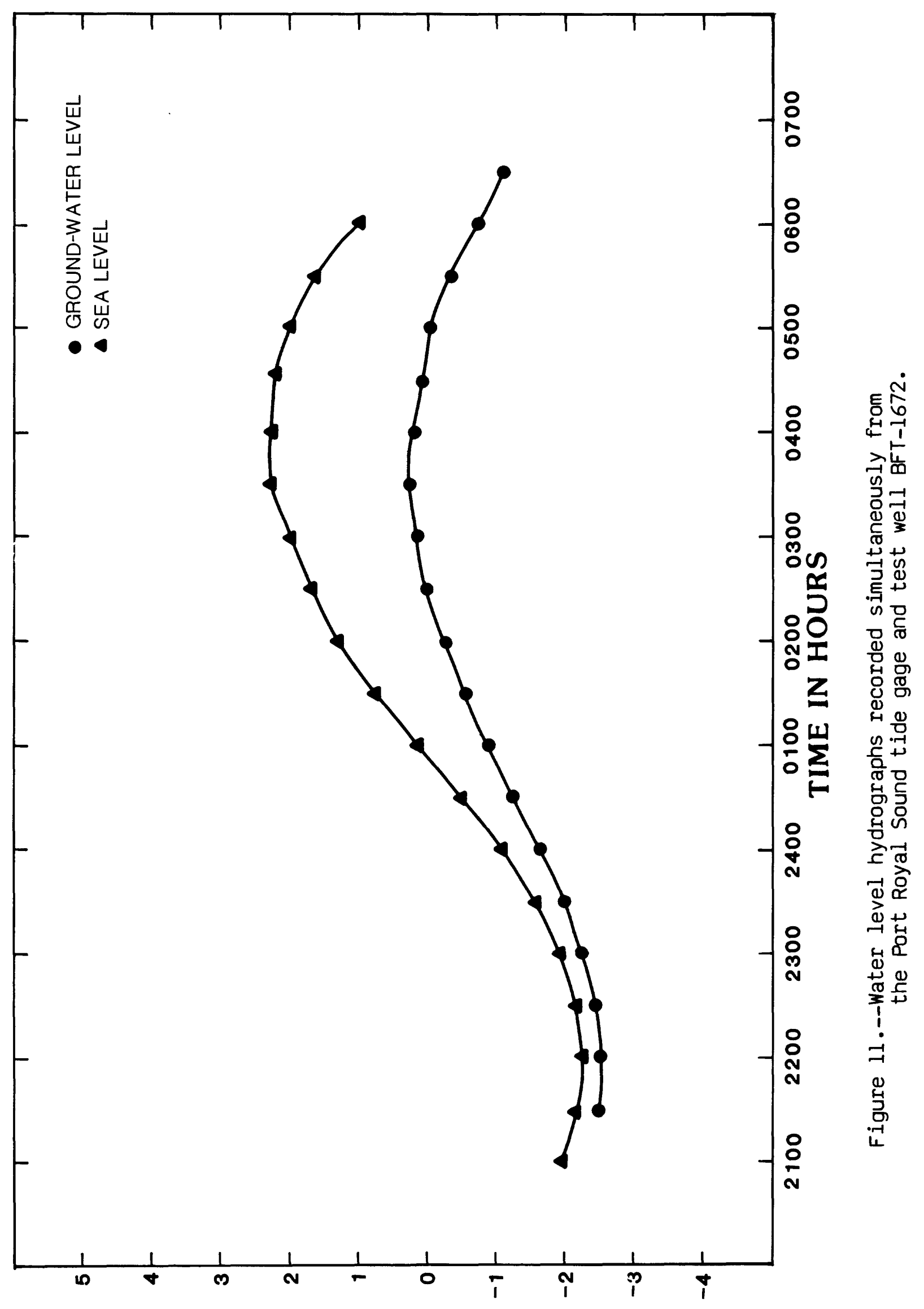

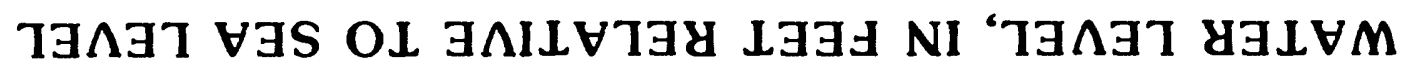




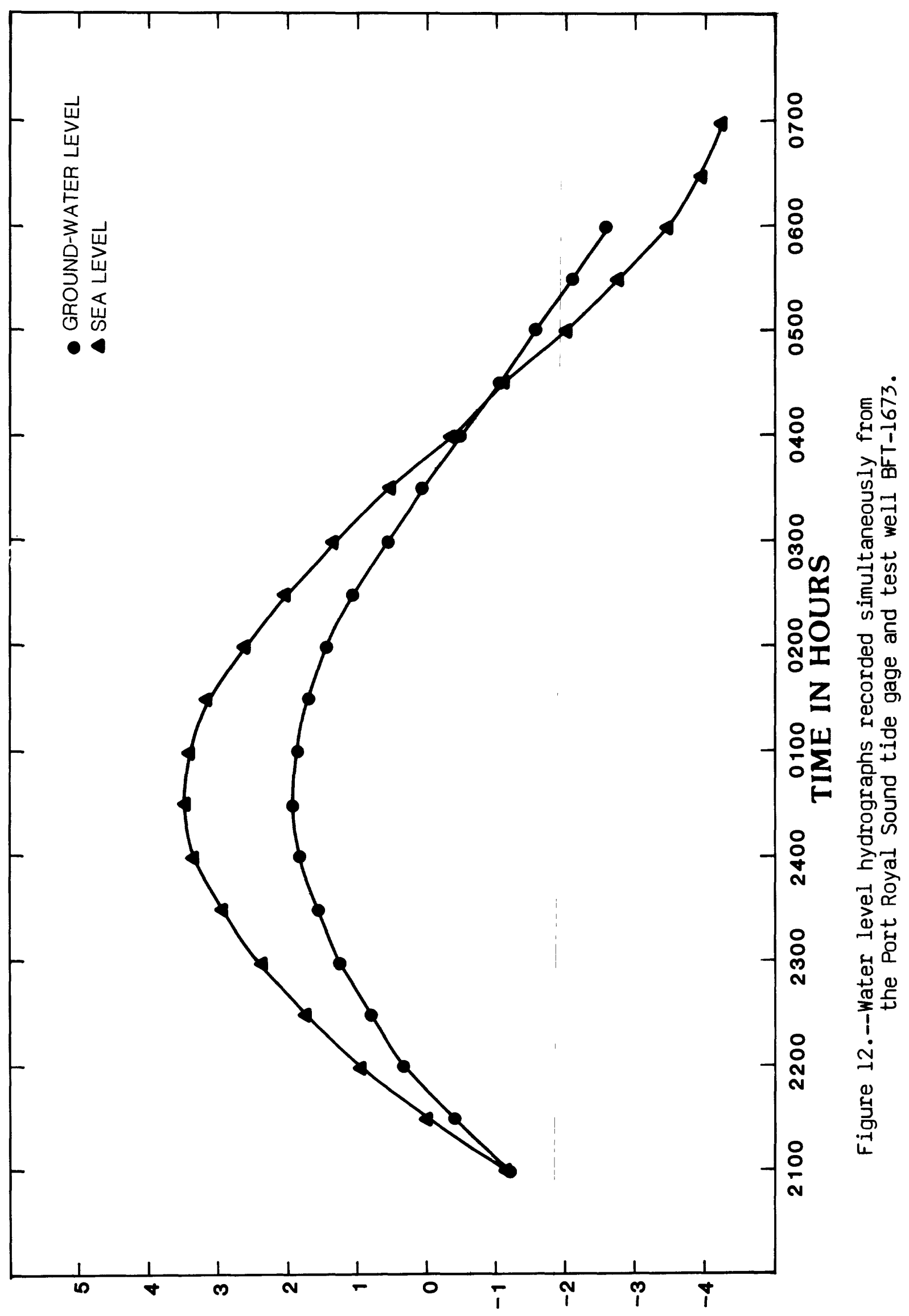

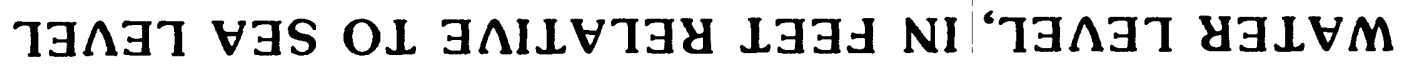




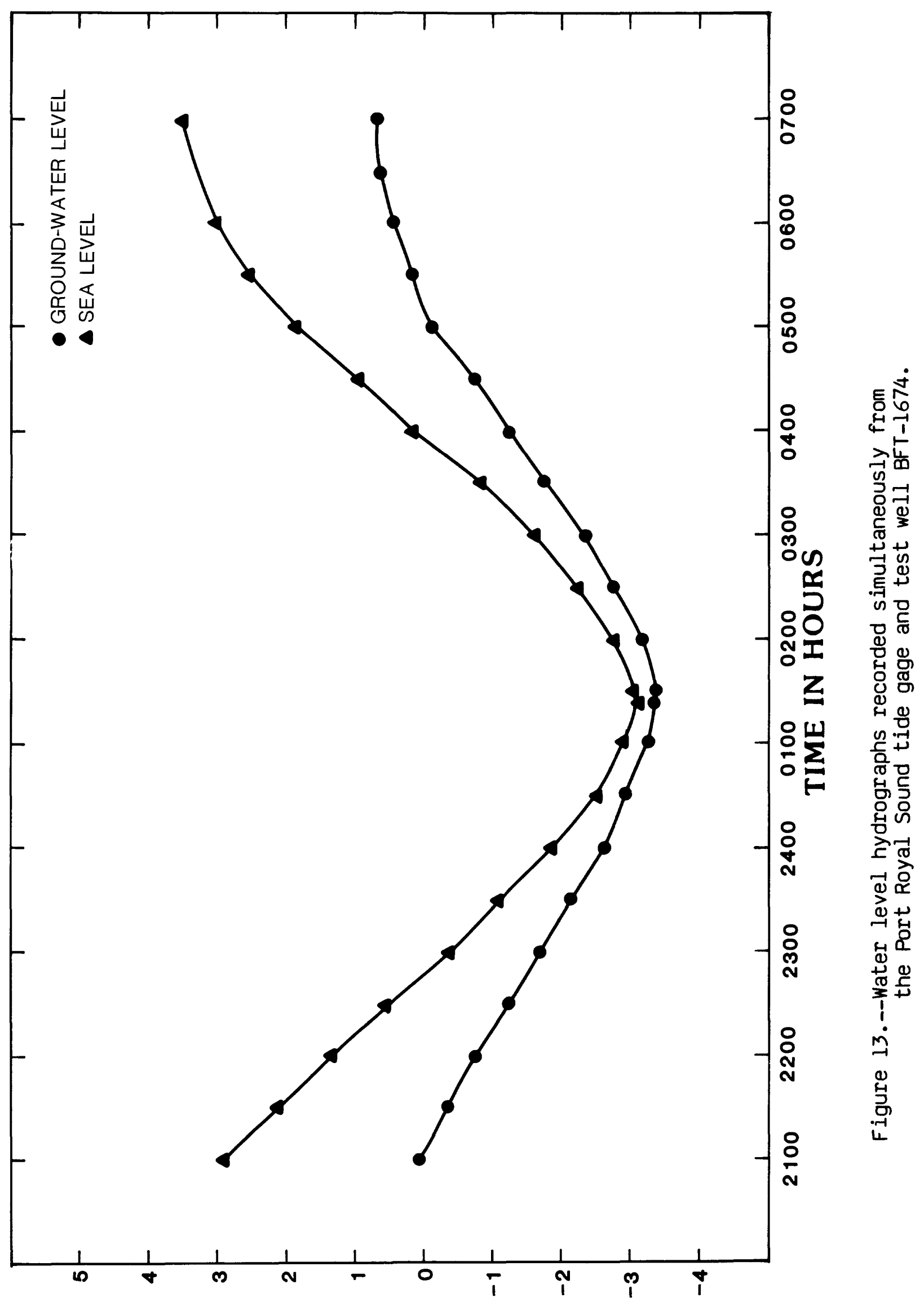

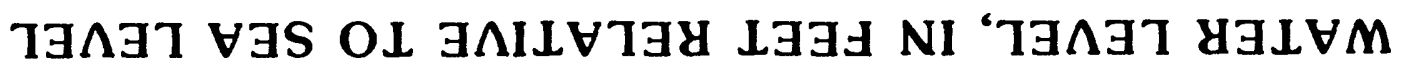




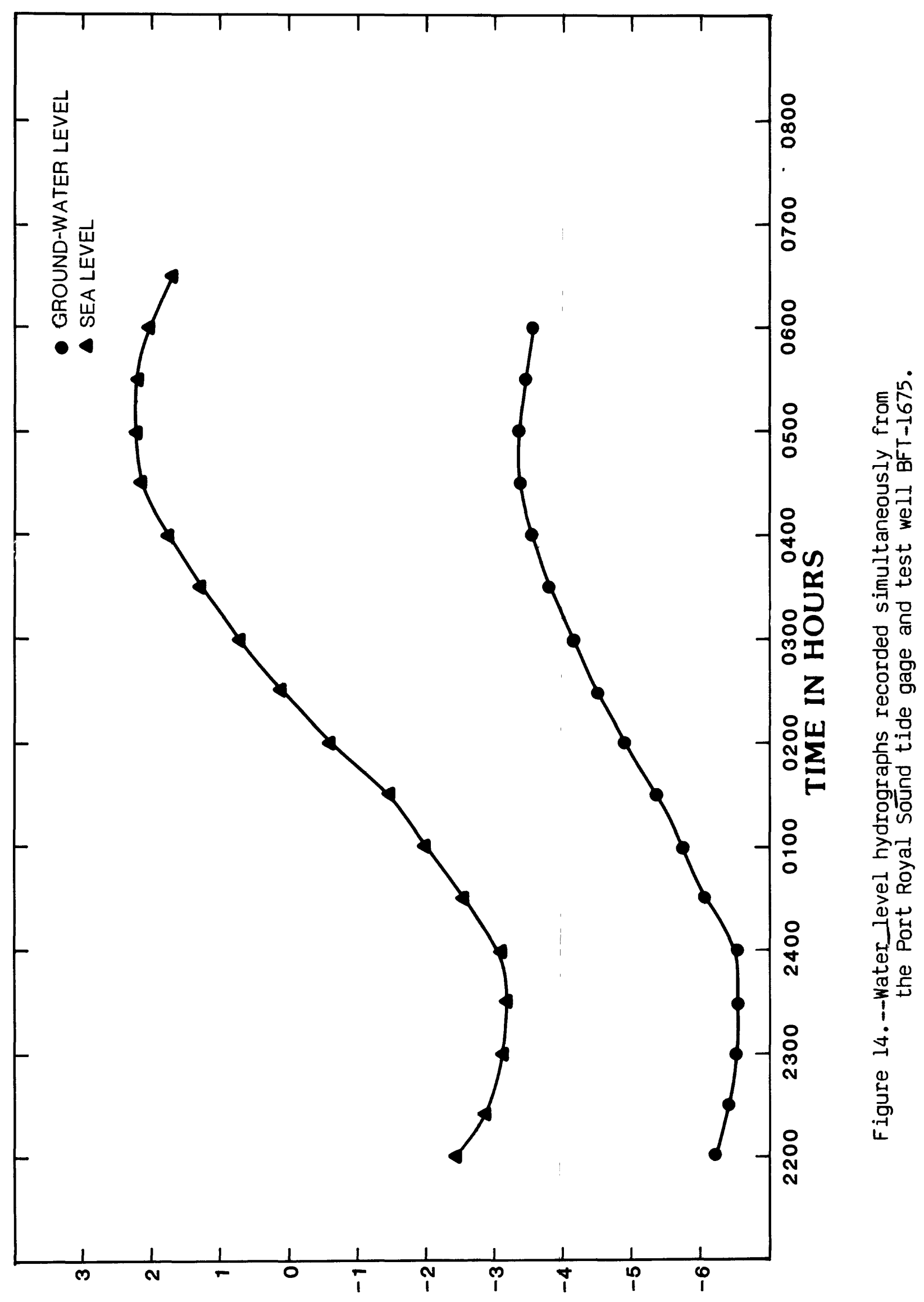

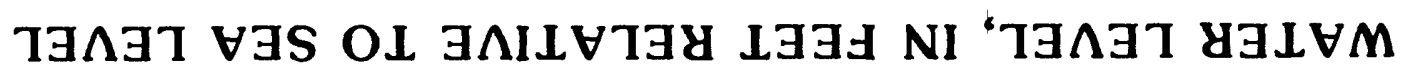




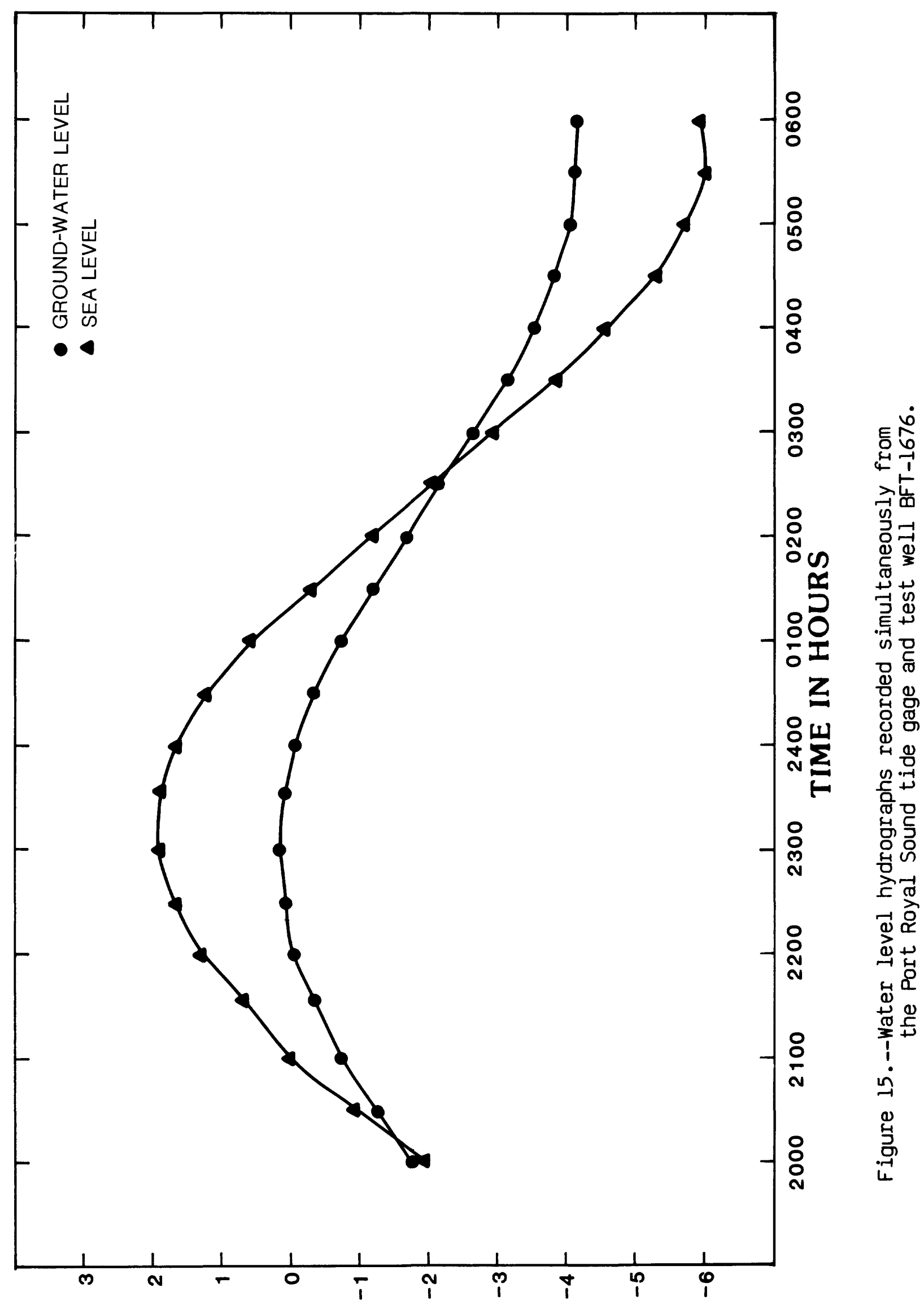

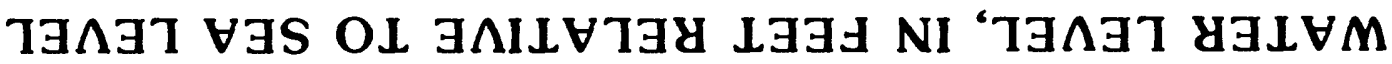




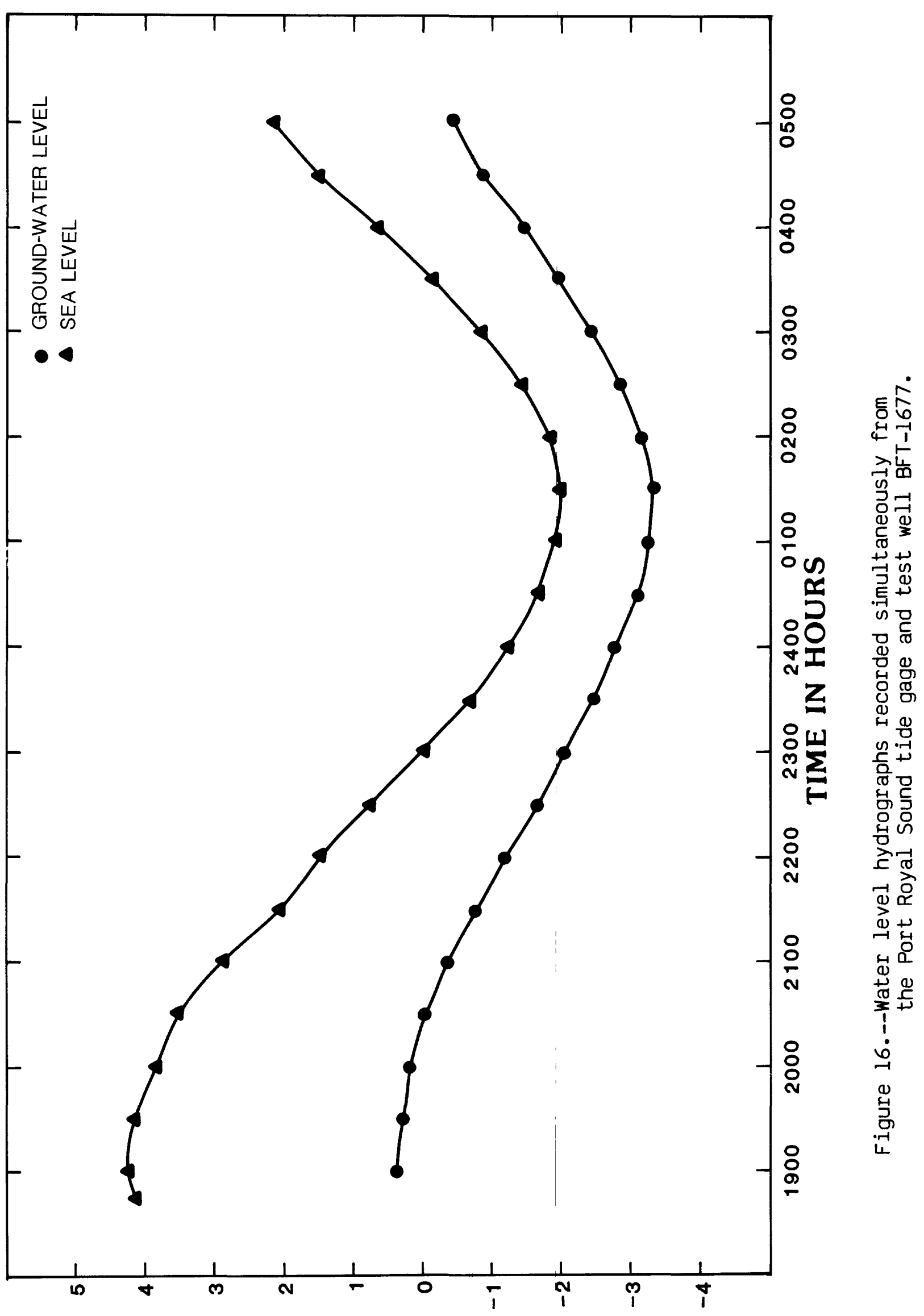

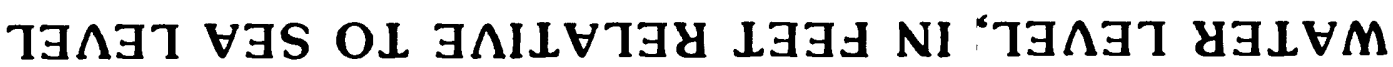




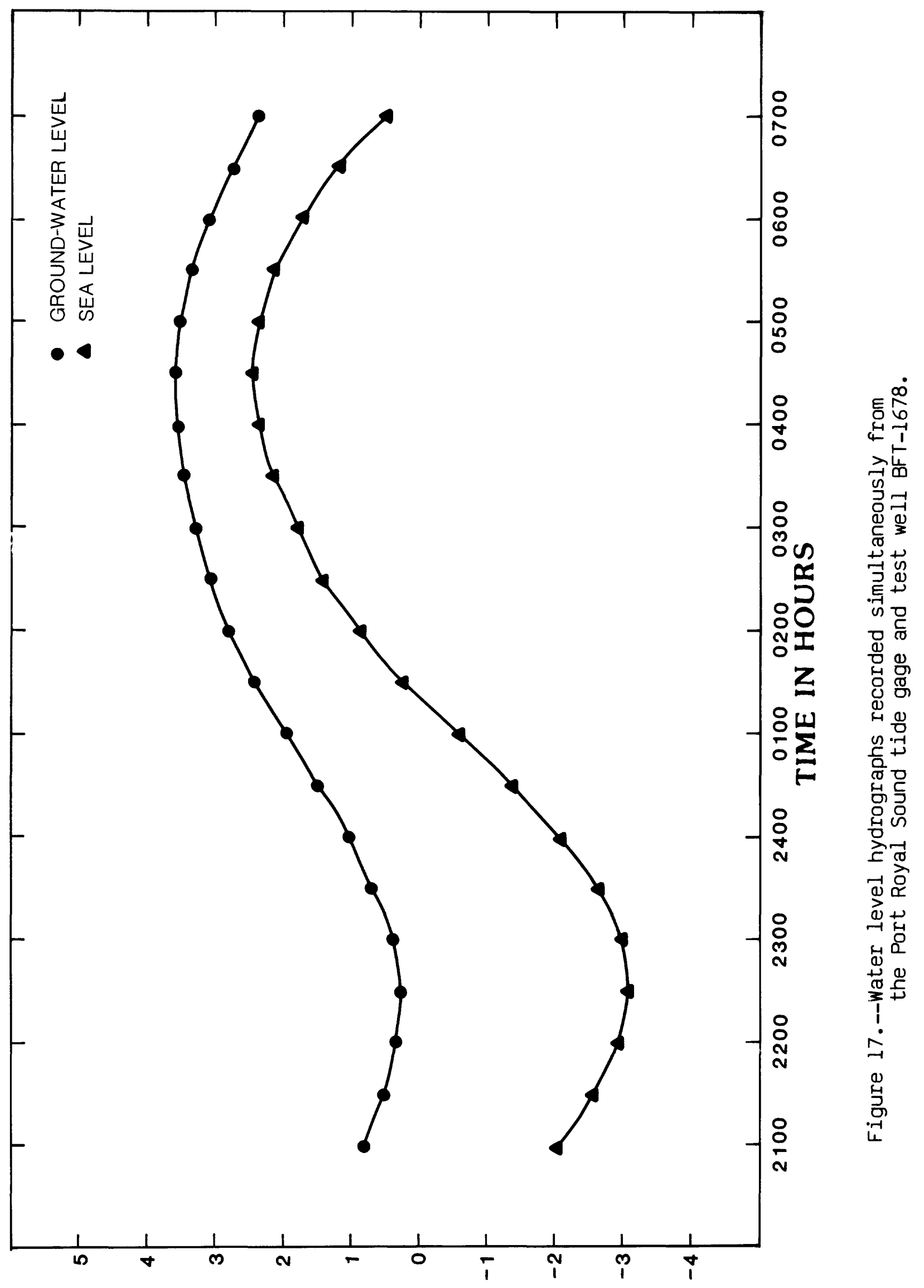

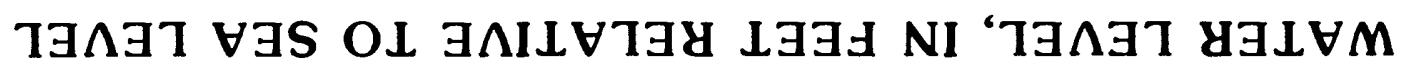




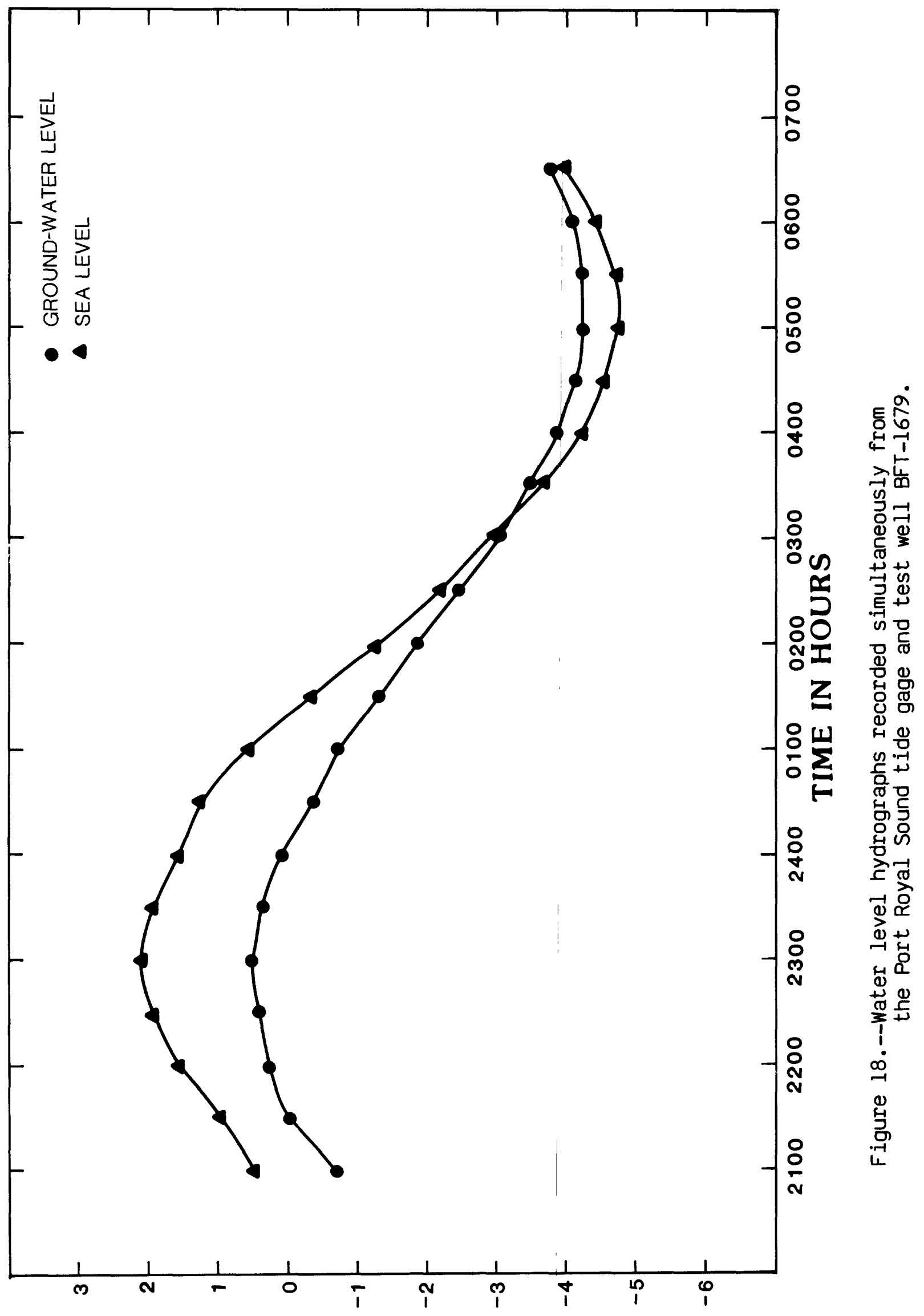

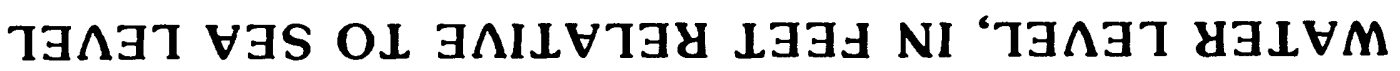




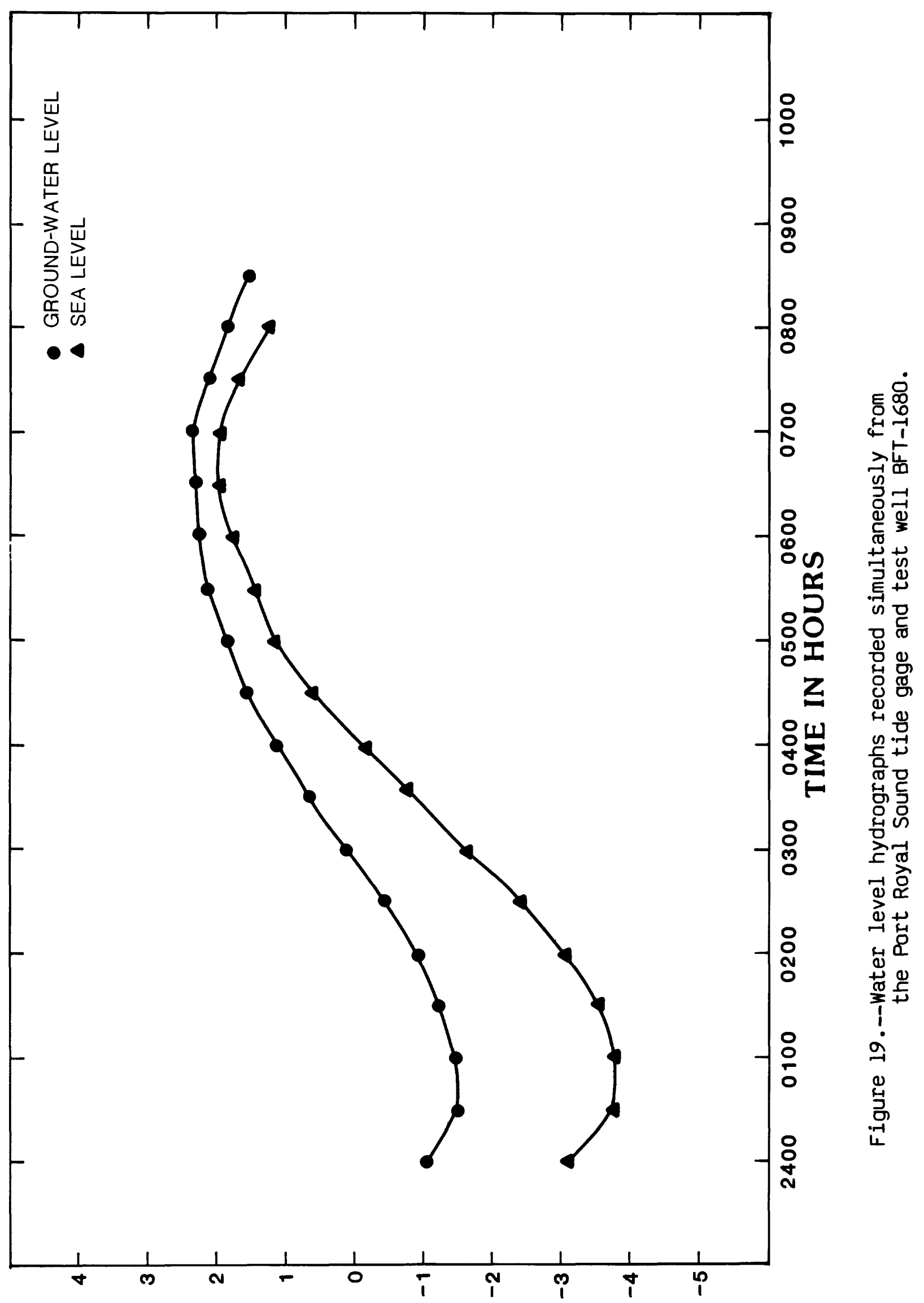

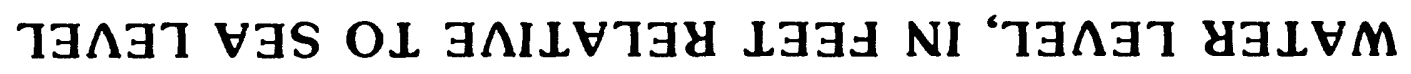


Table 1.--Well construction data

\begin{tabular}{|c|c|c|c|c|c|}
\hline Well & $\begin{array}{c}\text { North } \\
\text { Latitude }\end{array}$ & $\begin{array}{c}\text { West } \\
\text { Longitude }\end{array}$ & $\begin{array}{l}\text { Altitude } \\
\text { of top of } \\
\text { casing } \\
\text { (ft above } \\
\text { sea level) } \\
\pm 0.16\end{array}$ & $\begin{array}{l}\text { Altitude } \\
\text { of bottom } \\
\text { of casing } \\
\text { ( } \mathrm{ft} \text { below } \\
\text { sea level) }\end{array}$ & $\begin{array}{l}\text { Altitude } \\
\text { of bottom } \\
\text { of well } \\
\text { ( } \mathrm{ft} \text { below } \\
\text { sea level) }\end{array}$ \\
\hline
\end{tabular}

\begin{tabular}{|c|c|c|c|c|c|}
\hline $\begin{array}{l}\text { BFT-1672 } \\
\text { BFT-1673 } \\
\text { BFT-1674 } \\
\text { BFT-1675 } \\
\text { BFT-1676 } \\
\text { BFT-1677 } \\
\text { BFT-1678 } \\
\text { BFT-1679 } \\
\text { BFT-1680 }\end{array}$ & 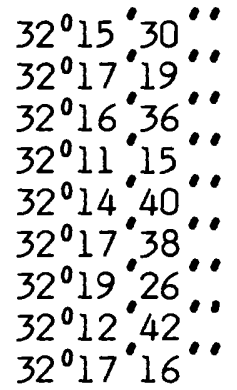 & 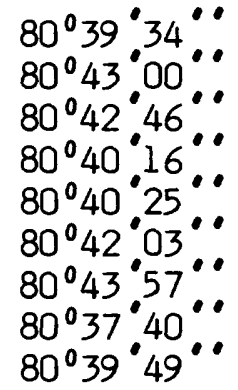 & $\begin{array}{l}10.76 \\
11.67 \\
15.45 \\
10.26 \\
13.25 \\
13.06 \\
11.16 \\
12.77 \\
14.28\end{array}$ & $\begin{array}{r}97.90 \\
83.93 \\
102.15 \\
91.34 \\
97.15 \\
91.84 \\
76.74 \\
102.35 \\
103.12\end{array}$ & $\begin{array}{l}211.44 \\
208.60 \\
174.30 \\
212.10 \\
211.00 \\
175.80 \\
191.70 \\
210.40 \\
217.50\end{array}$ \\
\hline
\end{tabular}

Table 2.--Lithologic descriptions of cores and cuttings from the Port Royal Sound test wells

\begin{tabular}{ll|}
\hline Well & Sample \\
interval & (ft below \\
& sea level)
\end{tabular}

\section{Cuttings}

BFT-1672 45.7 - 46.7 Sand, quartz, subangular to subrounded, coarse, yellow-gray ( 5 Y 7/2), shell fragments minor.

46.7 - 48.7 Sand, quartz, angular to sub-angular, medium, light olive-gray ( 5 Y $5 / 2$ ), shell fragments minor, phosphate minor.

48.7 - 49.7 Sand, quartz, subangular, coarse, medium light gray (N6), 30 percent shell fragments.

49.7 - 55.7 Sand, quartz, rounded to subrounded, medium light gray (N6), contains abundant mica flakes, wood fragments common, glauconitic, shell fragments minor. 
Table 2.--Lithologic descriptions of cores and cuttings from the Port Royal Sound test wells--Continued

\begin{tabular}{ll}
\hline \multirow{3}{*}{ Well } & Sample \\
& interval \\
& (ft below \\
& sea level)
\end{tabular}

\section{Core}

BFT-1672 55.7 - 56.7 Sand, quartz, subrounded, fine, medium gray (N5), (Continued) shell fragments minor, glauconitic.

$56.7-58.7$ As above, but sand is coarse.

58.7 - 59.7 Sand, quartz, subrounded, coarse to very coarse, greenish-gray (5GY 6/1), few shell fragments, abundant phosphate.

59.7 - 60.7 As above, but contains minor silt.

60.7 - 61.7 Sand, quartz, subrounded, coarse, olive gray (5Y $4 / 1$ ), few shell fragments.

61.7 - 63.2 Sand and pebbles quartz subangular, very coarse, grayish-yellow green ( $5 G Y 7 / 2$ ), abundant shell fragments, abundant very fine sand and silt, phosphate nodules.

63.2 - 65.7 Sand, quartz, subrounded, coarse, olive gray (5y 3/2), clay minor.

65.7 - 68.7 As above, but contains shell fragments and pebbles.

68.7 - 70 Sand, quartz, subangular, coarse, grayish-olive (10Y $4 / 2$ ), clay minor.

$70-71.7$ Silt and clay, olive gray $(5 Y 3 / 2)$.

71.7 - 73.7 Mixed layers of sand, quartz, subangular, grayish-olive (1OY $4 / 2$ ) and silty clay, olive gray (5Y $3 / 2)$.

73.7 - 76.7 As above, but dominantly silty clay.

76.7 - 80.3 As above, but clay and sand are present in equal amounts.

80.3 - 81.3 Silty clay with minor sand lenses, angular, coarse grayish-olive (10Y 4/2), calcareous. 
Table 2.--Lithologic descriptions of cores and cuttings from the Port Royal Sound test wells--Continued

\begin{tabular}{ll}
\hline Well & Sample \\
& interval \\
& (ft below \\
& sea level)
\end{tabular}

\section{Core}

BFT-1672 $81.3-84.1$ (Continued)

84.1 - 86.2 Silt and very fine sand, olive gray (5Y 3/2); abundant sand, quartz, coarse, calcareous.

86.2 - 87.9 As above, but more silt and clay.

87.9 - 98.1 Clay and silt, olive gray (5Y 3/2) and moderate sand, quartz, subrounded, medium, in some lenses sand exceeds clay in abundance.

98.1 - 99.2 Sand, quartz, rounded, fine, light gray (N7), in calcareous mud.

99.2 - 101.1 Limestone, fossiliferous, light gray (N7), abundant bryozoan fragments; minor sand, quartz, subangular fine.

101.1 - 102.8 Limestone, fossiliferous, pinkish-gray (5YK 8/1), dominantly bryozoans cemented with crystalline calcite, minor phosphate.

102.8 - 106 Limestone, fossiliferous, light gray (N7) bryozoans and pelecypods common, moderately well consolidated.

106 - 106.4 Limestone, fossiliferous, white (N9), composed of bryozoans and calcite crystals in calcareous mud.

106.4 - 119 Limestone, fossiliferbus, light gray (N7), composed of calcite-cemented bryozoan fragments. 
Table 2.--Lithologic descriptions of cores and cuttings from the Port Royal Sound test wells--Continued

\begin{tabular}{ll}
\hline Well & Sample \\
& interval \\
& (ft below \\
& sea level)
\end{tabular}

\section{Cuttings}

BFT-1672 119 - 142.3 Limestone, fossiliferous, light gray (N7);

(Continued)

predominantly fragments of bryozoans and pelecypods, minor calcite crystals.

142.3 - 157.3 As above, but bryozoans have larger pores and color

is very light gray (N8).

157.3 - 171.4 Limestone, fossiliferous, very light gray (N8), almost completely composed of bryozoan fragments and few pelecypod fragments, contains "cap-shaped" bryozoan.

171.4 - 177.3 As above, but light gray (N7).

177.3 - 192.3 As above, but slightly phosphatic.

192.3 - 215.4 Limestone, fossiliferous, light gray (N7), mostly bryozoans and some gastropod fragments, moderately glauconitic.

215.4 - 215.7 Silty clay, greenish-gray (5GY 6/1), calcareous.

BFT-1673 $35-41$ Sand, quartz, subangular, medium, greenish-gray (5GY 6/1), moderately phosphatic.

41 - 45 Sand, quartz, angular, medium, olive gray (5Y 4/1), abundant phosphate.

45 - 51 As above, but contains minor shell fragments, calcareous.

51 - 55 Sand, quartz, subangular, coarse, light olive gray (5Y 6/1), abundant phosphate, minor glauconite and shell fragments, calcareous.

$55-61$ As above, but less phosphate.

61 - 65 Sand, quartz, angular, very coarse, light gray (N7), minor glauconite and shell fragments, abundant coarse phosphate, calcareous. 
Table 2.--Lithologic descriptions of cores and cuttings from the Port

\begin{aligned} & \hline Well Sample \\ & interval \\ & (ft below \\ & sea level) \end{aligned}

\section{Cuttings}

BFT-1673 $65-71$ As above, but contains minor silt, greenish-gray (5GY

(Continued) $6 / 1$ ); pebble layer at $70.3^{\prime}$.

71 - 71.9 Sand, quartz, subangular, very coarse, olive gray ( $5 Y$ $4 / 1$ ), minor silt, phosphate, contains fragments of bryozoans, calcareous.

71.9 - 84.9 As above, but approximately 50 percent of sample composed of bryozoan fragments.

\section{Core}

84.9 - 87.2 Limestone fossiliferous, light gray (N7), very poorly consolidated, composed entirely of bryozoan fragments; at top large phosphate nodules up to $25 \mathrm{~mm}$ in long axis.

87.2 - 87.4 Marl, fragments of bryozoans in calcareous silt and clay matrix, light gray (N7).

87.4 - 93.9 Limestone fossiliferous, very light grey (N8), loosely consolidated, primarily composed of bryozoans but contains common pelecypods; breaks along planes of weaker material into layers $\frac{1}{2}$ in. to 2 in. thick.

93.9 - 95.4 As above, but all poorly consolidated.

95.4 - 95.8 Limestone, fossiliferous, light gray (N7), large round bryozoans and branching bryozoans, well consolidated.

95.8 - 100.8 As above, but unconsolidated.

100.8 - 105.8 Limestone fossiliferous, very light gray (N8), well consolidated; composed of bryozoans and pelecypods cemented with calcite.

105.8 - 110.8 As above, but very light gray (N8) and poorly consolidated.

110.8 - 111.3 Limestone, fossiliferous, light gray (N7), composed of very large $(20-30 \mathrm{~mm})$ bryozoan fragments with common pelecypod shells, well consolidated. 
Table 2.--Lithologic descriptions of cores and cuttings from the Port Royal Sound test wells--Continued

\begin{tabular}{ll}
\hline \multirow{3}{*}{ Well } & Sample \\
& interval \\
& (ft below \\
& sea level)
\end{tabular}

\section{Core}

BFT-1673 111.3 - 119.8 As above, but fossils are primarily branching (Continued) bryozoans and poorly consolidated.

119.8 - 122.3 Limestone, fossiliferous, light gray (N7), loosely consolidated, mostly bryozoans with few gastropods; breaks in thin plates ( $20 \mathrm{~mm}$ thick).

122.3 - 124.3 As above, but poorly consolidated.

124.3 - 125.8 Limestone, fossiliferous, light gray (N7) and light bluish gray (5 B $7 / 1$ ), dominantly pelecypods, well cemented with calcite.

\section{Cuttings}

125.8 - 141.1 Limestone, fossiliferous, light gray (N7), 50 percent bryozoans, remainder composed of pelecypods and gastropods with some crystalline calcite.

141.1 - 151.1 Limestone, fossiliferous, very light gray (N8), dominantly bryozoans, gastropods common, crystalline calcite minor.

151.1 - 161.1 As above.

161.1 - 171.1 Limestone, fossiliferous, light gray (N7), dominantly bryozoans, few gastropods and pelecypods, moderately glauconitic, better consolidated than above.

171.1 - 181.1 As above.

181.1 - 191.1 Limestone, fossiliferous, light gray (N7), fossils as above, less glauconite than above.

191.1 - 201.1 As above.

\section{Core}

201.1 - 208.6 Limestone, sandy, very fine, silty, greenish-gray (5G $6 / 1$ ), moderately galuconitic and phosphatic, few shell fragments. 
Table 2.--Lithologic descriptions of cores and cuttings from the Port Royal Sound test wells--Continued

\begin{tabular}{ll}
\hline \multirow{W}{*}{ Well } & Sample \\
& interval \\
& (ft below \\
& sea level)
\end{tabular}

\section{Cuttings}

BFT-1674 23.2 - 24.3 Sand, quartz, subrounded, medium, yellowish-gray (SY $7 / 2$ ), moderately phosphatic, muscovite minor.

$24.3-29.3$ Sand, quartz, subrounded, fine, light olive gray (5y $6 / 1)$, moderately phosphatic, muscovite minor, calcareous.

$29.3-44.3$ As above, but greenish-gray (5GY 6/1).

44.3 - 46.3 Sand, quartz, subangular, medium, greenish-gray (5GY $6 / 1$ ), few phosphate nodules, few wood fragments, calcareous.

46.3 - 48.8 Sand, quartz, subangular, coarse, olive gray (5Y 4/1), minor silt, moderately phosphatic.

48.8 - 56.3 Sand, quartz, subangular, medium olive gray ( $5 Y 4 / 1$ ), moderately phosphatic, minor silt, few shell

fragments.

56.3 - 59.5 Sand, quartz, angular, fine, and clay ( 30 percent), olive gray ( $5 Y 4 / 1)$, moderately phosphatic, muscovite minor, abundant $15 \mathrm{~mm}$ phosphate nodules at $58.1 \mathrm{ft}$, calcareous.

$59.5-60.3$ As above, but clay is 50 percent.

$60.3-61.8$ Same as $56.3-59.5$.

\section{Core}

61.8 - 68.3 Sand, quartz, subrounded, fine, olive gray (5Y $3 / 2$ ), moderate clay ( 30 percent), phosphate minor, muscovite minor, calcareous.

68.3 - 69 Sand, quartz, rounded, medium, light gray (N7) in matrix of poorly cemented calcite, phosphate minor.

$69-69.2$ Pebbles, quartz, angular, common clay and silt; sand quartz, angular, medium, olive gray ( $5 Y 4 / 1)$, common pebble-size phosphate nodules.

69.2 - 69.9 Sand, quartz, subangular, medium, olive gray ( $5 Y 4 / 1)$ and olive black ( 5 Y 2/1), minor clay and silt, phosphate minor. 
Table 2.--Lithologic descriptions of cores and cuttings from the Port Royal Sound test wells--Continued

\begin{tabular}{ll}
\hline Well & Sample \\
& interval \\
& (fT below \\
& sea level)
\end{tabular}

\section{Core}

BFT-1674 149.3 - 154.3 Limestone, fossiliferous, light gray (N7), mostly (Continued) bryozoans but pelecypods are common.

\section{Cuttings}

154.3 - 159.3 As above, but slightly glauconitic.

159.3 - 166.3 Limestone, fossiliferous, very light gray (N8), contains 50 percent bryozoans with remainder composed of gastropods and pelecypods, minor glauconite and phosphate.

166.3 - 174.3 As above, but rock is more cohesive.

\section{Core}

69.9 - 73.8 Sand, quartz, subangular, medium, light olive gray ( 5 Y $6 / 1$ ), weakly cemented with calcite, moderately phosphate.

73.8 - 74.3 As above, unconsolidated.

74.3 - 79.3 Sand, quartz, subrounded, very fine and medium, olive gray ( 5 Y $4 / 1$ ), weakly consolidated, moderately phosphatic, calcareous.

79.3 - 89.3 Sand, quartz, subangular, medium, olive black ( $5 Y$ $2 / 1$ ), abundant clay (50 percent), phosphate minor.

89.3 - 91.3 As above, but clay dominates (70 percent).

91.3 - 91.8 As above, but contains blocks of sand, quartz, subangular, medium, light gray (N7), in calcareous matrix.

$91.8-95.3$ Sand, quartz, subrounded, very fine, olive gray ( $5 Y$ $4 / 1$ ), moderately consolidated with calcite.

95.3 - 98.3 Clay, olive gray ( 5 Y $4 / 1$ ), and minor sand quartz, sub-angular, fine. 
Table 2.--Lithologic descriptions of cores and cuttings from the Port Royal Sound test wells--Continued

\begin{tabular}{ll}
\hline Well & Sample \\
& interval \\
& (ft below \\
& sea level)
\end{tabular}

\section{Cuttings}

BFT-1674 98.3 - 113.3 Limestone, fossiliferous, light gray (N7), dominantly (Continued) composed of bryozoan fragments.

113.3 - 123.3 As above, but contains pelecypod fragments.

123.3 - 133.3 As above, but color is pinkish-gray ( 5 YR 8/1) to very light gray (N8).

133.3 - 143.3 Limestone, fossiliferous, light gray (N7), composed primarily of bryozoans but few foraminifera and ostracoda.

143.3 - 149.3 As above, but pinkish-gray (5YR 8/1).

\section{Cuttings}

BFT-1675 10.7 - 17.2 Sand, quartz, angular, medium, medium gray (N5), abundant phosphate, common shell fragments, micaceous (muscovite), calcareous.

17.2 - 30.7 As above, but contains coarse sand and abundant shell fragments.

30.7 - 35.7 Sand, quartz, angular, coarse, dark greenish-gray (5GY 4/1), common phosphate, abundant shell fragments, common clay, calcareous.

35.7 - 41.7 As above, but clay is abundant.

\section{Core}

41.7 - 42.8 Clay, olive gray $(5 y 4 / 1)$, breaks into angular blocks, minor phosphate.

42.8 - 47.1 Sand, quartz, subangular, very coarse, greenish-gray ( $5 G Y$ 6/1), minor clay, minor phosphate, minor shell fragments.

47.1 - 49.1 Clay, olive gray (5Y $4 / 1$ ), breaks into angular blocks.

49.1 - 49.6 Phosphate rock, black (N1), very well consolidated, few gastropod fossils. 
Table 2.--Lithologic descriptions of cores and cuttings from the Port Royal Sound test wells--Continued

\begin{tabular}{ll}
\hline Well & Sample \\
& interval \\
& (ft below \\
& sea level)
\end{tabular}

\section{Core}

BFT-1675 49.6 - 52.5 Marl, clay, very light gray (N8); sand, quartz (Continued) rounded, coarse, medium gray (N5), abundant phosphate pebbles, calcareous.

52.5 - 55.3 Sandstone, calcareous; sand, quartz, subangular, medium, cemented with crystalline calcite, very light gray (N8), well consolidated, minor phosphate.

55.3 - 57.1 Marl; sand, quartz, subrounded, medium, olive gray (5Y 4/1); clay, olive gray $(5 Y 4 / 1)$, calcareous.

$57.1-63.1$ As $52.5-55.3$

63.1 - 66.6 Sandstone, calcareous; sand, quartz, rounded, medium; matrix is crystalline calcite, very light gray (N8), well consolidated, moderate phosphate.

66.6 - 67.1 As above, but calcite is not well cemented.

67.1 - 75.1 Sand, quartz, subangular, coarse, olive gray ( $5 Y$ 4/1), minor silt, minor phosphate.

75.1 - 77.1 As above, but contains common clay.

77.1 - 83.1 Sand, quartz, subangular, fine, dark greenish-gray ( $5 G Y 4 / 1$ ), clay moderate (30 percent), minor phosphate.

83.1 - 87.1 Sand, quartz, subangular, medium, dark greenish-gray (5GY 4/1), moderate clay (25 percent), calcareous.

87.1 - 87.7 Clay, dark greenish-gray (5GY 4/1), large phosphate nodules ( 5 to $50 \mathrm{~mm}$ ).

87.7 - 87.8 Phosphate rock, black (NI), well consolidated.

87.8 - 88.5 Limestone, fossiliferous, very light gray (N8) and pale greenish-yellow (10Y $8 / 2$ ), contains large fenestrate bryozoans, very hard and well consolidated.

88.5 - 95 Marl, branching bryozoan fragments and sand quartz subrounded, fine, in a matrix of calcareous mud, yellowish gray ( 5 Y $7 / 2)$. 
Table 2.--Lithologic descriptions of cores and cuttings from the Port Royal sound test wells--Continued

\begin{tabular}{ll}
\hline Well & Sample \\
& interval \\
& (ft below \\
& sea level)
\end{tabular}

Core

BFT-1675 95 - 97.1 Silty clay and calcite, white (N9), few sand grains, (Continued) subangular, medium.

\section{Cuttings}

97.1 - 102.1 Limestone, fossiliferous, very light gray (N8), composed of bryozoans and some crystalline calcite, few phosphate nodules.

102.1 - 112.1 Limestone, fossiliferous, white (N9), contains fenestrate and branching bryozoans, and few cap-shaped bryozoans.

112.1 - 122.1 As above, but no cap-shaped bryozoans, and contains few coarse calcite sand grains.

122.1 - 132.1 Limestone, fossiliferous, very light gray (N8) dominantly composed of branching bryozoans, few pelecypods and fenestrates.

132.1 - 142.1 As above, but contains some gastropods.

142.1 - 152.1 Limestone, fossiliferous, white (N9), mostly composed of branching bryozoans, few foraminifera.

152.1 - 162.1 As above, but no foraminifera.

162.1 - 172.1 Limestone, fossiliferous, very light gray (N8), composed of branching bryozoans and some fenestrate forms, few foraminifera, pyrite minor.

172.1 - 182.1 Limestone, fossiliferous, white (N9), composed of branching and fenestrate bryozoans, few pelecypod fragments.

182.1 - 192.1 As above, but contains minor pyrite.

192.1 - 202.1 As above, but slightly glauconitic and phosphate.

202.1 - 212.1 Limestone, fossiliferous, very light gray (N8), contains fenestrate and branching bryozoans, and up to 40 percent pelecypods, minor glauconite, phosphate, and pyrite. 
Table 2.--Lithologic descriptions of cores and cuttings from the port Royal Sound test wells--Continued

\begin{tabular}{ll}
\hline Well & Sample \\
& interval \\
& (ft below \\
& sea level)
\end{tabular}

\section{Cuttings}

BFT-1676 49.7 - 50.7 Sand, quartz, subrounded, coarse, dark gray (N3); angular fragments of chert, coarse, brownish-gray (5YR 4/1); limestone, crystalline, white (N9); abundant coarse angular phosphate, some pyrite coatings on phosphate grains.

50.7 - 51.2 As above, but contains very fine calcite sand and few fragments of rose quartz, angular, fine, pale pink (5RP 8/2).

51.2 - 56.7 Sand, quartz, subrounded, coarse, light gray (N7); chert fragments, few, angular, brownish-gray (5YR 4/1), minor limestone, crystalline, white (N9); abundant medium subrounded phosphate.

56.7 - 57.7 As above, but no chert and phosphate grains are rounded.

57.7 - 59.7 Sand, quartz, coarse and fine, subrounded, olive gray ( $5 Y 4 / 1)$; clay, minor, olive gray ( $5 Y 4 / 1)$; phosphate minor.

59.7 - 62.7 As above, but contains no fine sand.

\section{Core}

62.7 - 67.7 Sand, quartz, subangular, coarse, olive gray (5Y 4/1); clay common; phosphate common.

67.7 - 71.7 Sand, quartz, subangular, coarse, greenish-gray (5GY $6 / 1$ ), in matrix of calcareous silt and clay, phosphate common.

71.7 - 73.7 Sand, quartz, subrounded coarse, grayish-olive (10Y $4 / 2$ ), moderate clay ( 30 percent), phosphate minor.

73.7 - 74.1 Sand, quartz, subrounded, medium, very light gray (N8); calcite sand, very fine, white (N9).

74.1 - 82.1 Sand, quartz, subangular, fine, olive gray ( 5 Y 4/1); moderate clay (20 percent), phosphate minor. 
Table 2.--Lithologic descriptions of cores and cuttings from the Port Royal Sound test wells--Continued

\begin{tabular}{ll}
\hline Well & Sample \\
interval & ft below \\
sea level) & \\
\hline & Core \\
\hline
\end{tabular}

BFT-1676 82.1 - 90.2 As above, but clay is slightly more abundant (Continued) (25-30 percent).

90.2 - 91.7 Sand, quartz, subangular, fine, olive gray ( $5 Y 4 / 1)$, clay abundant ( 50 percent), moderate phosphate.

91.7 - 92.4 As above, but contains up to 60 percent of clay.

92.4 - 93.8 Sand, quartz, subangular, fine, and clay, olive gray (5Y 4/1); calcite mud, fossiliferous, contains foraminifera and bryozoans in upper part sand dominates in lower part calcite mud dominates.

93.8 - 94.2 Large phosphate nodules (10mm).

94.2 - 95.2 Limestone, crystalline, white (N9), very well consolidated, non-porous, contains few bryozoans.

95.2 - 108.5 Limestone, fossiliferous, very light gray (N8), composed of small fragments of branching bryozoans, phosphate minor.

\section{Cuttings}

108.5 - 117.7 Limestone, fossiliferous, pinkish-gray (5YR 8/1), composed of branching bryozoans with few fenestrate forms, few foraminifera.

117.7 - 129.7 As above, but color is light gray (N7).

129.7 - 139.7 Limestone, fossiliferous, very light gray (N8), bryozoans, many slender branching forms and fenestrate, pelecypod fragments common.

139.7 - 149.7 As above.

149.7 - 159.7 Limestone, fossiliferous, very light gray (N8), branching and few fehestrate bryozoans, few pelecypods, phosphate minor, pyrite minor.

159.7 - 169.7 As above, but is dominantly composed of branching bryozoans and contains no pelecypods.

169.7 - 179.7 Limestone, fossiliferous, light gray (N7), composed of branching bryozoans and few pelecypods, pyrite minor. 
Table 2.--Lithologic descriptions of cores and cuttings from the Port Royal Sound test wells--Continued

\begin{tabular}{ll}
\hline Well & Sample \\
interval \\
$(\mathrm{ft}$ below \\
sea level)
\end{tabular}

\section{Cuttings}

BFT-1676 179.7 - 189.7 As above, but contains common pelecypods, few (Continued) gastropods.

$189.7-200$ As above, but contains minor glauconite.

\section{Core}

$200-201$ Limestone, fossiliferous, white (N9), casts and molds of pelecypods and gastropods in crystalline calcite matrix, very well consolidated.

\section{Cuttings}

$201-211$ Limestone, fossiliferous, light gray (N7), composed of branching bryozoans with few fenestrate forms, polecypod fragments common, glauconite common.

\section{Cuttings}

BFT-167798.1 - 108.1 Limestone, fossiliferous, very light gray (N8), mostly branching bryozoans and few pelecypod fragments.

108.1 - 118.1 As above, but has minor pyrite.

118.1 - 128.1 Limestone, fossiliferous, light gray (N7), contains branching and fenestrate bryozoans, few gastropods, few foraminifera, pyrite minor.

128.1 - 138.1 As above, but pelecypod fragments are common.

138.1 - 148.1 Limestone, fossiliferous, light gray (N7), bryozoans branching and fenestrate, pelecypods common, pyrite common.

148.1 - 158.1 As above, but contains minor crystalline calcite and minor galuconite.

158.1 - 168.1 As above, but more glauconitic and phosphatic. 
Table 2.--Lithologic descriptions of cores and cuttings from the Port Royal Sound test wells--Continued

\begin{tabular}{|c|c|}
\hline Well & $\begin{array}{l}\text { Sample } \\
\text { interval } \\
\text { (ft below } \\
\text { sea level) }\end{array}$ \\
\hline
\end{tabular}

\section{Core}

BFT-1677 168.1 - 171.8 Limestone, soFT, clayey, pale blue green (5BG 7/2) (Continued) fossiliferous, bryozoans and pelecypods few, abundantly glauconitic and phosphatic.

171.8 - 175.8 Limestone, fossiliferous, SoFT, clayey, very light gray (N8), contains bryozoan fragments, abundant glauconite and phosphate.

\section{Cuttings}

BFT-1677 46.6 - 51.6 Sand, quartz, subangular medium, light gray (N7); crystalline calcite, white (N9); chert minor, angular, fine, grayish-red (1OR 4/2), phosphate common.

51.6 - 56.6 As above, but contains some shell fragments and less calcite.

56.6 - 66.6 Sand, quartz, subrounded, coarse, medium gray (N5), minor crystalline calcite, white (N9), chert minor, subrounded, fine, grayish-red (IOR 4/2), phosphate common.

66.6 - 68.6 As above, but contains minor muscovite.

68.6 - 78.6 Sand, quartz, subangular, coarse, olive gray (5Y $4 / 1$ ), chert minor, rounded, fine, light brown (5YR $5 / 6)$, phosphate minor, calcareous. 
Table 2.--Lithologic descriptions of cores and cuttings from the Port Royal Sound test wells--Continued

\begin{tabular}{ll}
\hline Well & Sample \\
interval \\
(ft below \\
sea level)
\end{tabular}

\section{Core}

BFT-1677 78.6 - 82.8 Sand, quartz, subrounded, coarse, light olive gray (Continued)

(5Y 6/1), clay (25 percent), light gray (N7), calcareous, chert minor, rounded, fine, light brown (5YR 5/6); moderately phosphatic.

82.8 - 89.1 Sand, quartz, angular, medium, olive black (5Y 2/1); clay ( 30 percent) olive black ( 5 Y $2 / 1$ ), chert minor rounded, fine, light brown ( 5 YR $5 / 6$ ), moderate phosphate.

89.1 - 93.4 Limestone, fossiliferous, light gray (N7), contains fenestrate and branching bryozoans, pyrite minor.

93.4 - 94.1 As above, but contains common pelecypods.

\section{Cuttings}

94.1 - 98.1 Limestone, fossiliferous, light gray (N7); dominantly branching bryozoans and few fenestrates.

BFT-1678 40.6 - 50.6 Sand, quartz, subangular, coarse, olive gray (5Y 4/1), limestone minor, crystalline, white (N9), phosphate common.

50.6 - 62.6 Sand, quartz, subangular, coarse, medium dark gray N4), calcite-cemented sandstone minor, and angular medium, very light gray (N8), phosphate minor.

\section{Core}

62.6 - 62.7 Gravel, rounded quartz, chert, and phosphate.

62.7 - 63.2 Clay, dark greenish-gray (5GY 4/1), sand, fine, minor. 
Table 2.-- Lithologic descriptions of cores and cuttings from the port Royal Sound test wells (Continued)

\begin{tabular}{ll}
\hline Well & Sample \\
& interval \\
& (ft below \\
& sea level)
\end{tabular}

\section{Core}

BFT-1678 63.2 - 67.6 Sand, quartz, angular, fine, dark greenish-gray (5GY (Continued) $4 / 1$ ), moderate clay, minor chert, rounded, fine, light brown (5YR 5/6), weakly consolidated, calcareous.

67.6 - 70.1 Limestone, soft, sandy, light gray (N7), sand, very fine, abundant, chert, fine, rounded, light brown (5YR 5/6), minor.

70.1 - 70.6 As above, but color is olive gray $(5 Y 4 / 1)$.

70.6 - 72.1 Sand, quartz, subangular, fine and coarse, clay, abundant, olive gray ( 5 Y $4 / 1)$, moderate phosphate, coarse, rounded, calcareous.

72.1 - 76.4 Crystalline limestone, light gray (N7), fossiliferous, contains pelecypods and bryozoans; top $0.5 \mathrm{FT}$ is phosphatic, well consolidated.

\section{Cuttings}

76.4 - 78.6 As above.

78.6 - 86.4 Limestone, fossiliferous, very light gray (N8), composed of bryozoans, branching and fenestrate.

86.4 - 96.4 As above, but color ranges to pinkish-gray (5YR $8 / 1$ ), branching bryozoans dominant.

96.4 - 101.7 Limestone, fossiliferous, white (N9), composed of fenestrate bryozoans, well cemented, pyrite minor.

101.7 - 111.7 Limestone, fossiliferous, very light gray (N8), contains abundant bryozoans, pelecypods and gastropod casts; fossils are coated and cemented with fine crystalline calcite, phosphate minor.

111.7 - 116.7 Limestone, fossiliferous, light gray (N7), abundant bryozoan and pelecypod fragments, moderately well cemented. 
Table 2.-- Lithologic descriptions of cores and cuttings from the Port Royal Sound test wells (Continued)

\begin{tabular}{ll}
\hline \multirow{W}{*}{ Well } & Sample \\
& interval \\
& (ft below \\
& sea level)
\end{tabular}

\section{Core}

BFT-1678 116.7 - 123.9 As above.

(Continued)

123.9 - 126.4 Limestone, fossiliferous, very light gray (N8), fossils are dominantly pelecypods with abundant gastropod fragments and shells, well consolidated.

\section{Cuttings}

126.4 - 141.7 Limestone, fossiliferous, light gray (N7), contains branching and fenestrate bryozoans, glauconite and phosphate minor.

141.7 - 151.7 As above, but glauconite is abundant.

151.7 - 161.7 Limestone, fossiliferous, light gray (N7), dominantly branching bryozoans and few fenestrates, few pelecypods, moderate glauconite.

161.7 - 171.7 Limestone, fossiliferous, very light gray (N8), branching and fenestrate bryozoans, pelecypods, and gastropods common, glauconite minor.

171.7 - 181.7 As above.

181.7 - 191.7 Limestone, fossiliferous, very light gray (N8), dominantly composed of branching bryozoans, few pelecypods and fenestrate bryozoans, pyrite minor, moderate glauconite.

\section{Cuttings}

BFT-1679 38.8 - 42.3 Sand, quartz, angular, coarse, very light gray (N8); abundant pelecypod shells and shell fragments, few bryozoans; moderate phosphate, mica (muscovite) minor.

42.3 - 52.3 As above, but contains abundant clay (30 percent), medium bluish gray (5B 5/1), phosphate abundant.

52.3 - 56.0 Sand, quartz, angular, coarse, light gray (N7), common shell fragments, phosphate abundant. 
Table 2.--Lithologic descriptions of cores and cuttings from the Port

Royal Sound test wells--Continued

\begin{tabular}{ll}
\hline Well & Sample \\
& interval \\
& (ft below \\
& sea level)
\end{tabular}

\section{Core}

BFT-1679 56.0 - 60.0 Clay, olive gray (5Y 3/2); sand, quartz, fine (30 percent), phosphate abundant.

60.0 - 64.8 Clay, olive black ( 5 Y 2/1); sand, quartz, very fine; chert, fine, rounded, light brown (5 YR 6/4); phosphate abundant.

$64.8-65.8$ As above.

65.8 - 66.8 Clay, olive gray (5Y 3/2), minor sand (10 percent), quartz, very fine, abundant phosphate, chert minor, fine, rounded, light brown (5YR 6/4).

66.8 - 68.3 Clay, olive gray ( 5 Y 3/2); sand, quartz, fine subangular; chert, fine, rounded, light brown (5YR $6 / 4$ ), phosphate abundant, calcareous.

68.3 - 69.6 Sandstone, sand, quartz, subangular, medium in matrix of well-cemented, fine, calcareous sand, medium light gray (N6).

69.6 - 74.3 As above, but calcite sand is weakly cemented.

74.3 - 78.3 Sand, quartz, subangular, fine, clay, olive black (5Y $2 / 1$ ), phosphate minor.

78.3 - 84.8 Sand, quartz, subangular coarse greenish-gray (5GY $6 / 1$ ), clay minor, phosphate minor, some layers of material like $(69.3$ - 74.3) mixed in.

84.8 - 92.3 As above, but contains more clay.

92.3 - 99.8 Sand, quartz, subangular coarse and medium, clay (30 percent), olive gray ( 5 Y $4 / 1)$, phosphate minor.

99.8 - 105.7 As above.

105.7 - 110.8 Limestone, fossiliferous, very light gray (N8), contains abundant pelecypods and fenestrate bryozoans, well consolidated. 
Table 2.--Lithologic descriptions of cores and cuttings from the Port Royal Sound test wells--Continued

\begin{tabular}{ll}
\hline Well & Sample \\
& interval \\
& (ft below \\
& sea level) \\
\hline
\end{tabular}

Cuttings

BFT-1679 110.8 - 120.4 Limestone, fossiliferous, very light gray (N8), (Continued) composed of branching bryozoan fragments and few fenestrate bryozoans.

120.4 - 130.4 As above, but color ranges to pinkish gray ( 5 YR 8/1).

130.4 - 140.4 Limestone, fossiliferous, light gray (N7), contains large fragments of fenestrate bryozoans, few branching bryozoans, moderately consolidated.

140.4 - 150.4 Limestone, fossiliferous, white (N9), composed of fenestrate and branching bryozoans.

150.4 - 160.4 Limestone, fossiliferous, light gray (N7), contains large fragments of fenestrate bryozoans, few pelecypods and branching bryozoans.

160.4 - 170.4 Limestone, fossiliferous, very light gray (N8), dominantly fenestrate and branching bryozoans, few gastropods and pelecypods, pyrite minor.

170.4 - 180.4 As above, but gastropods are absent.

180.4 - 190.4 Limestone, fossiliferous, very light gray (N8), composed of well-cemented bryozoans and pelecypods, few "cap-shaped" bryozoans, pyrite minor.

190.4 - 200.4 As above, but has minor glauconite and phosphate.

200.4 - 210.4 Limestone fossiliferous, light gray (N7), contains fenestrate bryozoans, few pelecypods, gastropods, and branching bryozoans; galuconite and phosphate abundant.

\section{Cuttings}

BFT-1680 15.7 - 25.7 Sand, quartz, subangular, coarse, light olive gray (5Y 6/1), shell fragments abundant; phosphate minor.

25.7 - 30.7 Sand, quartz, angular, coarse, medium light gray (N6), abundant shell fragments and wood fragments, mica flakes (muscovite) common, phosphate minor. 
Table 2.--Lithologic descriptions of cores and cuttings from the Port Royal Sound test wells--Continued

\begin{tabular}{ll}
\hline Well & Sample \\
& interval \\
& (ft below \\
& sea level)
\end{tabular}

\section{Cuttings}

BFT-1680 30.7 - 45.9 Sand, quartz, subangular, medium, light gray ( $5 Y$ (Continued) 4/1), phosphate minor.

45.9 - 53.5 Sand, quartz, subrounded, coarse, olive gray ( $5 Y$ 4/1), phosphate minor.

\section{Core}

53.5 - 56.7 Sand, quartz, subangular, coarse and very fine, light olive gray ( 5 Y $6 / 1$ ); phosphate minor, few gastropods.

56.7 - 60.0 As above, but contains 30 percent clay.

60.0 - 62.2 Sand, quartz, angular, coarse, light gray (N7), minor chert, rounded, medium, light brown ( 5 YR 5/6).

62.2 - 63.4 As above, but sand is in a matrix of calcareous clay, very light gray (N8).

63.4 - 64.4 Pebbles and sand, quartz, rounded, very coarse, greenish-gray ( $5 G Y 6 / 1$ ), minor chert, rounded, fine, light brown (5YR 5/6), calcareous.

64.4 - 65.4 Sand, quartz, subrounded, coarse, olive gray (5Y $4 / 1)$, moderate chert, rounded, fine, light brown (5YR 5/6), moderate calcareous clay, very light gray (N8).

65.4 - 73.3 As above, but lacks calcareous matrix.

73.3 - 80.7 Sand, quartz, subangular, medium, clay (40 percent), olive gray ( $5 Y 4 / 1)$, moderate fine sand, minor chert as above.

80.7 - 89.0 Sand, quartz, subangular, medium, clay (30 percent), olive gray (5Y $4 / 1)$, phosphate minor.

89.0 - 92.7 As above.

92.7 - 95.7 Sand, quartz, subangular, fine and clay (50 percent), olive gray ( $5 Y 4 / 1)$, phosphate moderate.

95.7 - 97.7 As above. 
Table 2.--Lithologic descriptions of cores and cuttings from the Port Royal Sound test wells--Continued

\begin{tabular}{ll}
\hline Well & Sample \\
interval \\
(ft below \\
sea level)
\end{tabular}

\section{Core}

BFT-1680 97.7 - 99.7 Limestone, soft, sandy, fine, very light gray (N8). (Continued)

99.7 - 106.9 Limestone, fossiliferous, very light gray (N8), clayey (10 percent), composed of slender branching bryozoans, few very coarse quartz grains (cave in).

106.9 - 117.1 Limestone, fossiliferous, very light gray (N8), composed of fenestrate bryozoans and pelecypods.

\section{Cuttings}

117.1 - 127.8 Limestone, fossiliferous, very light gray (N8), contains fenestrate and branching bryozoans, few pelecypods; pyrite minor.

127.8 - 137.8 As above, but contains few "cap-shaped" bryozoans.

137.8 - 147.8 Limestone, fossiliferous, very light gray (N8), branching and fenestrate bryozoans, pyrite minor.

147.8 - 157.8 Limestone, fossiliferous, light gray (N7), abundant pelecypod shells, bryozoans, minor glauconite.

157.8 - 167.8 As above, but contains gastropod casts.

167.8 - 177.8 Limestone, fossiliferous, very light gray (N8), composed of branching and fenestrate bryozoans, few pelecypod fragments, moderate glauconite, phosphate minor.

177.8 - 187.8 As above, but glauconite coats almost all grains and few gastropods. 
Table 2.--Lithologic descriptions of cores and cuttings from the port Royal Sound test wells--Continued

\begin{tabular}{ll}
\hline Well & $\begin{array}{l}\text { Sample } \\
\text { interval } \\
\text { (ft below } \\
\text { sea level) }\end{array}$ \\
\hline BFT-1680 187.8 - 197.8 As above, but light gray (N7). \\
$\begin{aligned} \text { (Continued) } \\
197.8-207.8 \text { Limestone, fossiliferous, light gray (N9), contains } \\
\text { bryozoans (branching and fenestrate), few pelecypod } \\
\text { fragments, phosphate minor, glauconite minor. }\end{aligned}$
\end{tabular}

\section{Core}

207.8 - 208.3 Limestone, soft, sandy, clayey; light olive gray (5Y $6 / 1$ ), fossiliferous, contains branching bryozoans and pelecypods, abundantly phosphatic and glauconitic.

208.3 - 217.5 Sand, calcite, subrounded, medium greenish-gray (5GY $6 / 1)$, moderately phosphatic and glaucontic. 


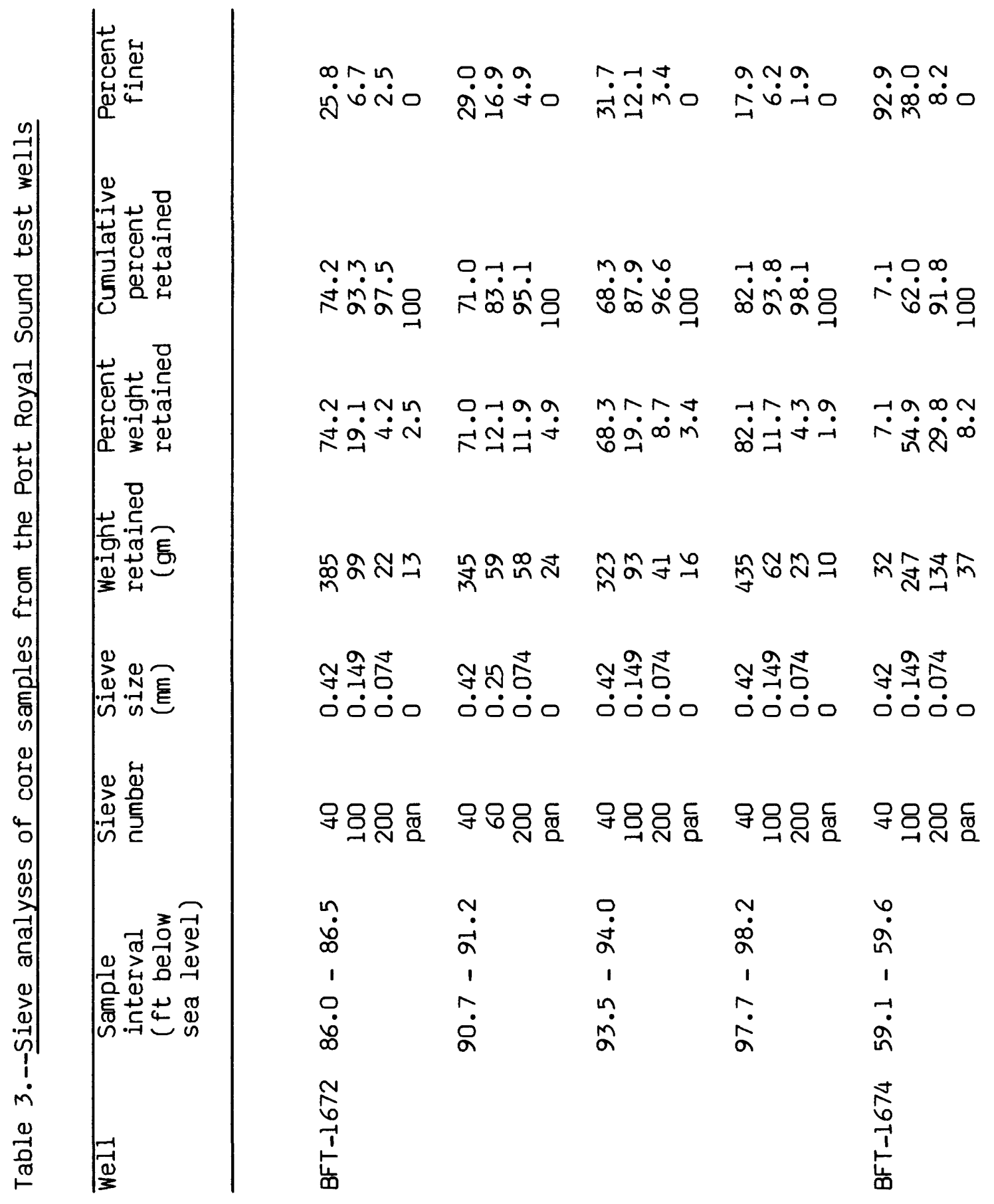




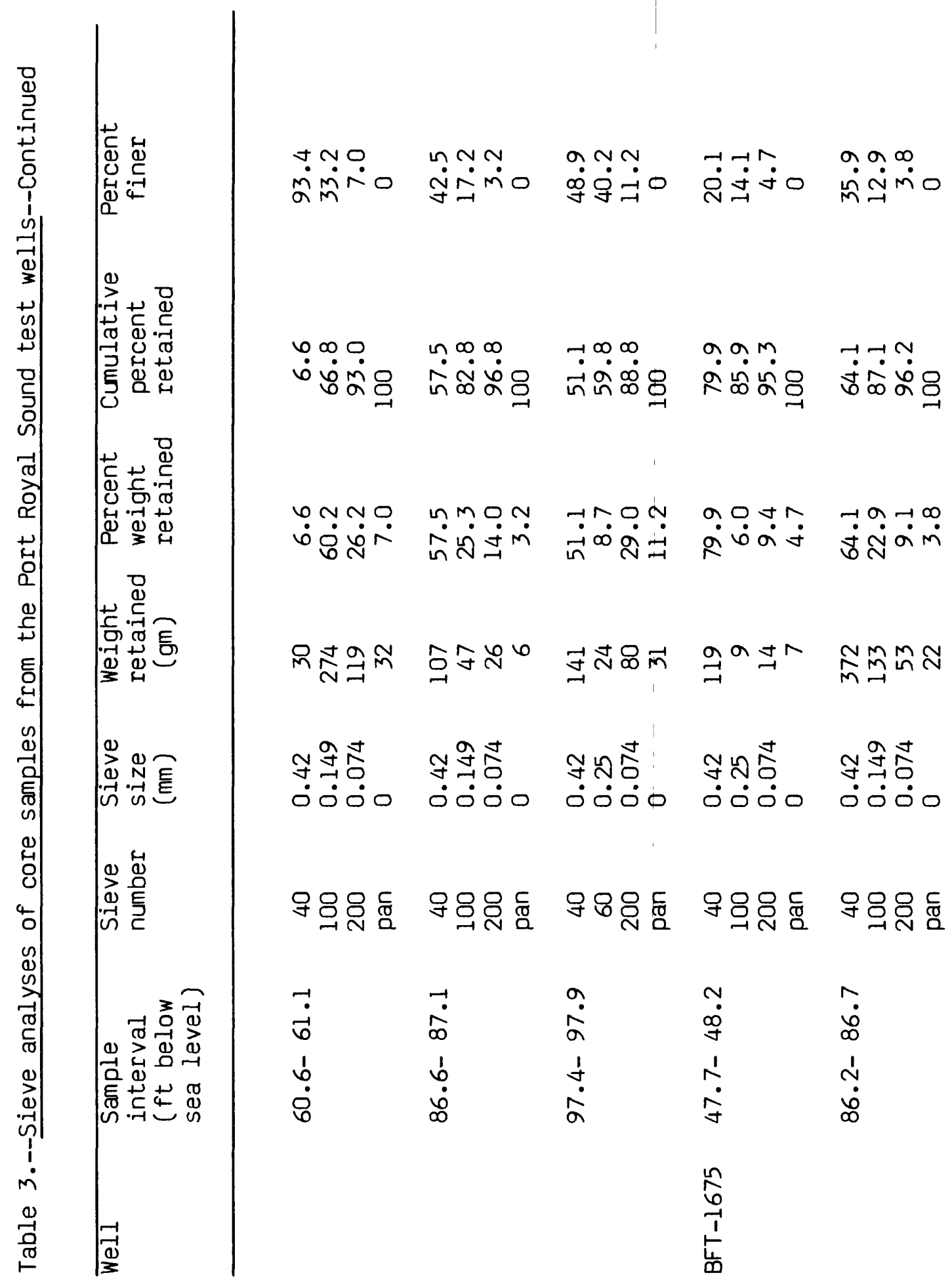




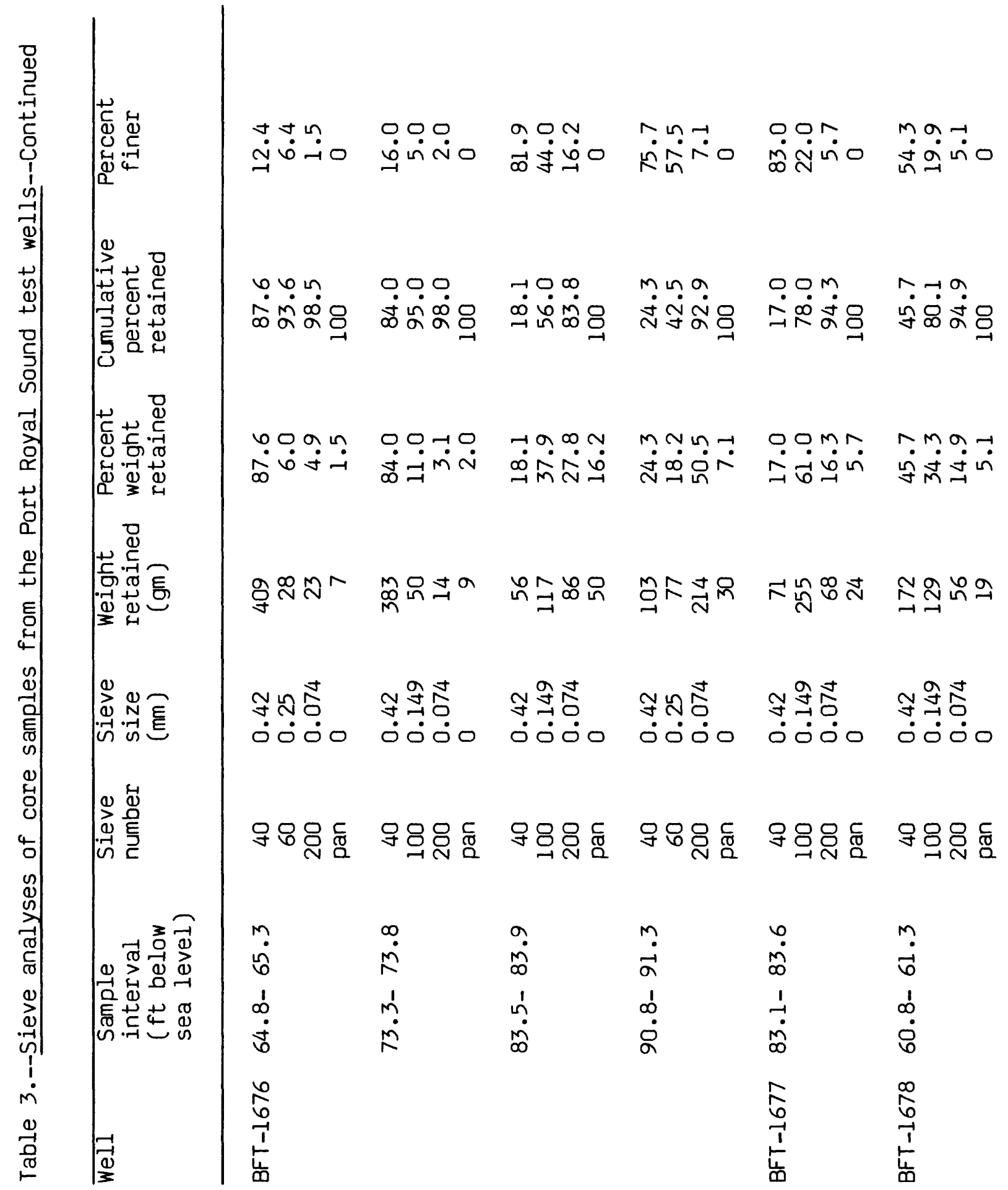




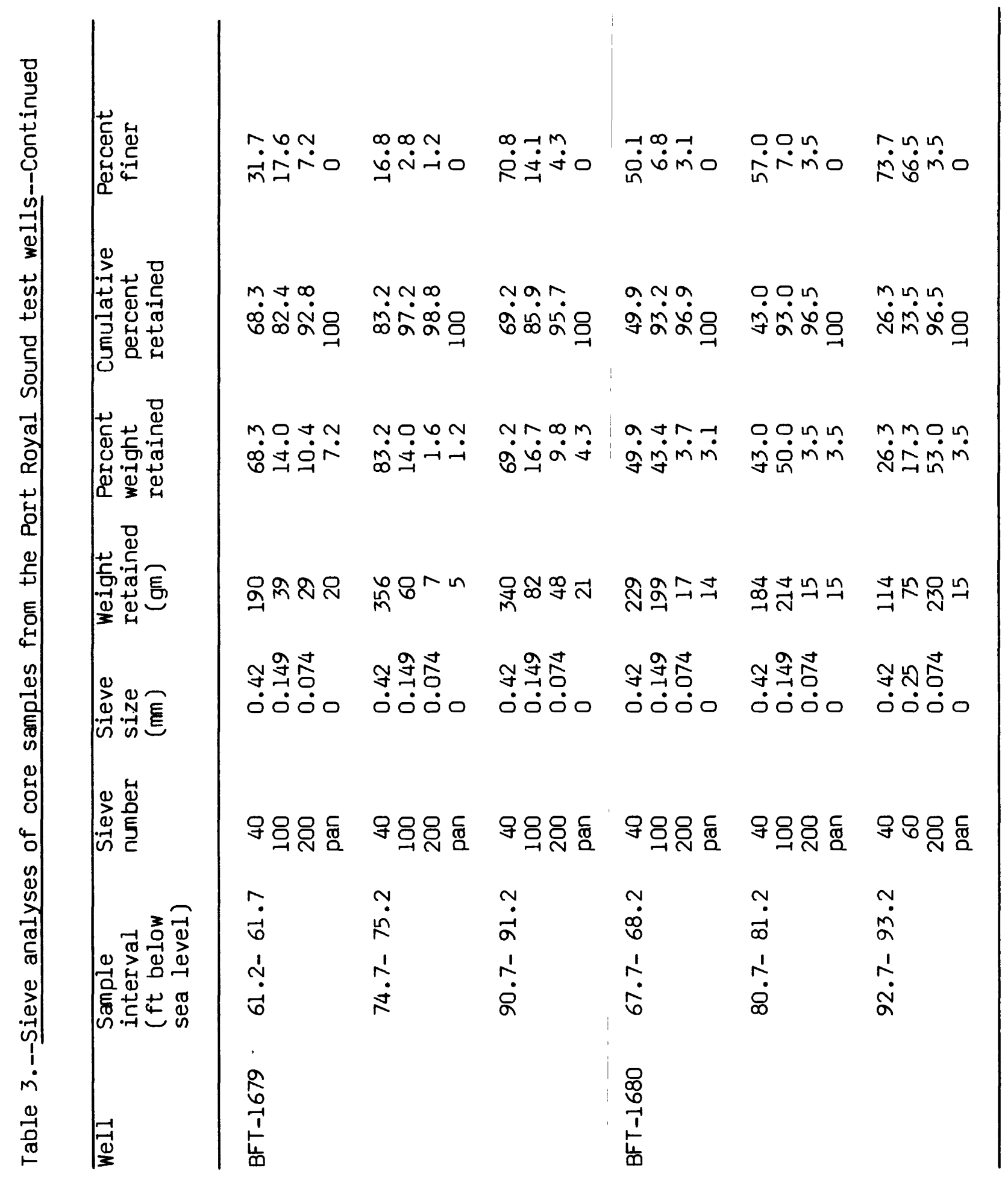




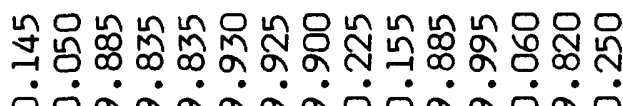

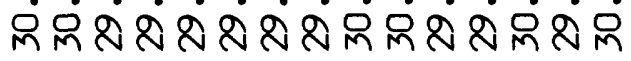

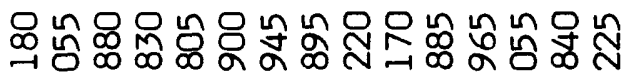

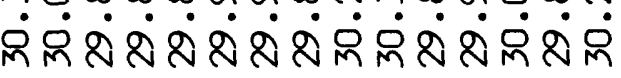

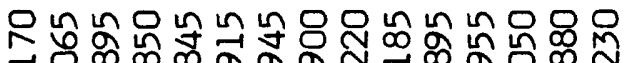
ఈ

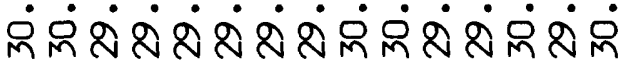

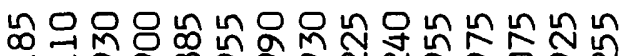

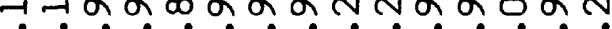

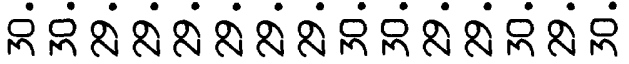

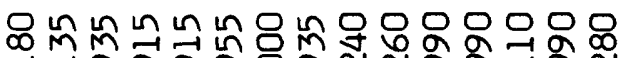

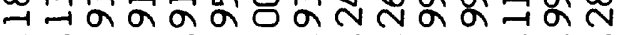

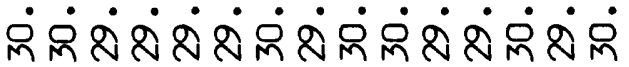

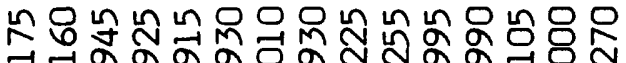

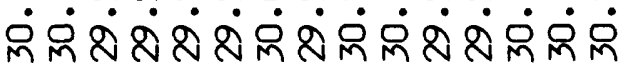

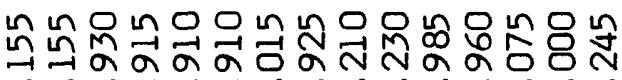

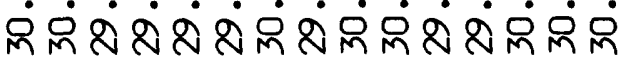

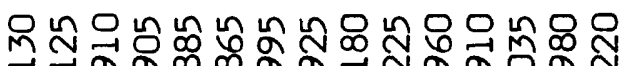

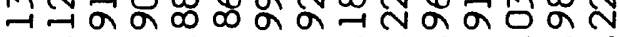
ப்

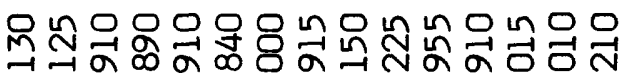

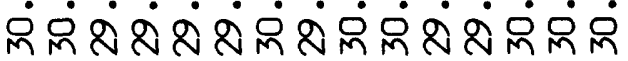

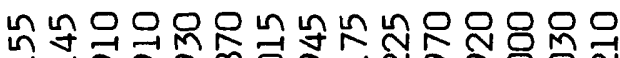

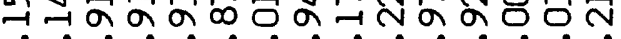

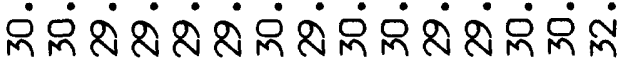

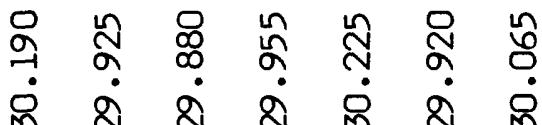

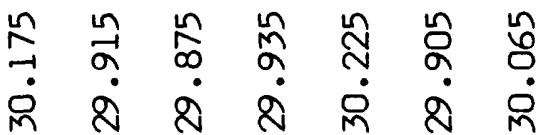

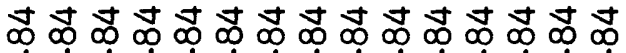

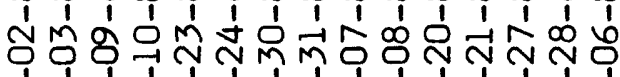
øó 
Table 5.--Falling head permeameter test results and gravimetric porosity data for selected cores from the Port Royal Sound test wells

\begin{tabular}{|c|c|c|c|}
\hline Well & $\begin{array}{c}\text { Sample interval } \\
\text { (feet below } \\
\text { sea level) }\end{array}$ & $\begin{array}{l}\text { Hydraulic } \\
\text { conductivity } \\
\text { (ft per day) }\end{array}$ & $\begin{array}{l}\text { Porosity } \\
\text { (percent) }\end{array}$ \\
\hline BFT-1672 & $\begin{array}{l}86.0-86.5 \\
90.7-91.2 \\
93 \cdot 5-94.0 \\
98 \cdot 7-99.2\end{array}$ & $\begin{array}{l}5.7 \times 10^{-3} \\
5.7 \times 10^{-3} \\
1.4 \times 10^{-2} \\
7.1 \times 10^{-2}\end{array}$ & $\begin{array}{l}41 \\
39 \\
-- \\
--\end{array}$ \\
\hline$B F T-1674$ & $\begin{array}{l}59 \cdot 1-59.6 \\
60.6-61.1 \\
86 \cdot 6-87.1 \\
97.4-97.9\end{array}$ & $\begin{array}{l}1.1 \times 10^{-2} \\
8.5 \times 10^{-3} \\
2.3 \times 10^{-1} \\
5.7 \times 10^{-4}\end{array}$ & $\begin{array}{l}44 \\
43 \\
38 \\
64\end{array}$ \\
\hline BFT-1675 & $\begin{array}{l}47.7-48.2 \\
86.2-86.7\end{array}$ & $\begin{array}{l}2.8 \times 10^{-3} \\
5.7 \times 10^{-3}\end{array}$ & $\begin{array}{l}72 \\
47\end{array}$ \\
\hline BFT-1676 & $\begin{array}{l}64 \cdot 8-65.3 \\
73 \cdot 3-73 \cdot 8 \\
83 \cdot 5-83.9 \\
90 \cdot 8-91.3\end{array}$ & $\begin{array}{l}2.8 \times 10^{-3} \\
3.1 \\
3.4 \times 10^{-2} \\
5.7 \times 10^{-3}\end{array}$ & $\begin{array}{l}45 \\
41 \\
48 \\
66\end{array}$ \\
\hline BFT-1677 & $83.1-83.6$ & $8.5 \times 10^{-3}$ & 45 \\
\hline BFT-1678 & $60 \cdot 8-61 \cdot 3$ & $1.2 \times 10^{-3}$ & -- \\
\hline BFT-1679 & $\begin{array}{l}61 \cdot 2-61 \cdot 7 \\
74 \cdot 7-75 \cdot 2 \\
90 \cdot 7-91 \cdot 2\end{array}$ & $\begin{array}{l}4.2 \times 10^{-4} \\
4.2 \times 10^{-1} \\
6.2 \times 10^{-2}\end{array}$ & $\begin{array}{l}-- \\
50 \\
44\end{array}$ \\
\hline $\mathrm{BFT}-1680$ & $\begin{array}{l}55 \cdot 2-55.7 \\
67.7-68.2 \\
80.2-80.7 \\
90.7-93.2\end{array}$ & $\begin{array}{l}2.8 \times 10^{-3} \\
8.5 \times 10^{-2} \\
2.0 \times 10^{-1} \\
2.0 \times 10^{-1}\end{array}$ & $\begin{array}{l}-- \\
45 \\
44 \\
35\end{array}$ \\
\hline
\end{tabular}




\begin{tabular}{|c|c|c|c|c|c|c|c|c|c|}
\hline 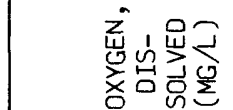 & ๑. .๑. & $\tilde{\sim} \tilde{\sim} \leadsto$ & $0.0 .-1$. & . & 0.999 & $\because \because \backsim$ ฯ & 0.0 .0$. & $\circ$. & יִ צִ \\
\hline 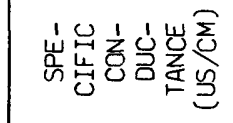 & 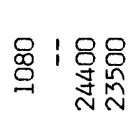 & 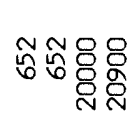 & 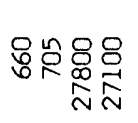 & 莳㣽芯怘 & 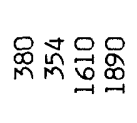 & 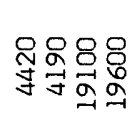 & 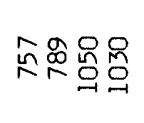 & 员号品 & 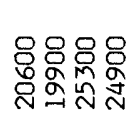 \\
\hline 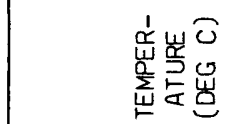 & $\ddot{\dot{N}}|\overrightarrow{\tilde{N}}|$ & $\mid \stackrel{n}{\mid} \underset{n}{\text { N }}$ & 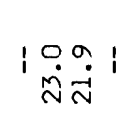 & $\begin{array}{l}0 M m \\
\dot{\Sigma} \dot{\sim} \\
\text { Nं }\end{array}$ & 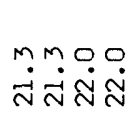 & 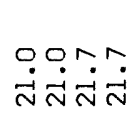 & $\begin{array}{l}\infty \\
\dot{i} \\
\dot{N}\end{array}$ & $\stackrel{\circ}{\dot{\sim}} \dot{\sim}$ & $\begin{array}{l}\text { åñ } \\
\dot{\alpha} \dot{\tilde{N}} \dot{\vec{N}}\end{array}$ \\
\hline 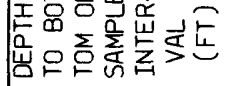 & 으으⿱츄 & 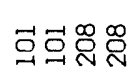 & 品寻寺去 & 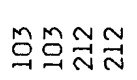 & 刍刍全 & ลัล゚ & œ & 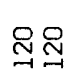 & $\stackrel{\Xi}{\exists} \underset{\sim}{*} \stackrel{\infty}{\sim}$ \\
\hline 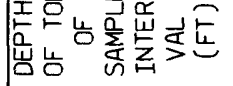 & 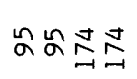 & 品员足足 & 험엄요모 & œ œ $\underset{\infty}{\infty} \underset{-\infty}{\infty} \underset{-1}{\infty}$ & 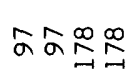 & ㅊํํํํำ & ำลีコี & $\stackrel{\infty}{\infty}$ & 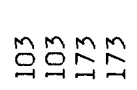 \\
\hline 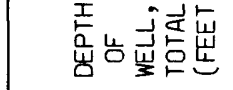 & $\exists \equiv \vec{\Xi} \vec{N}$ & 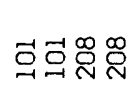 & 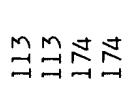 & 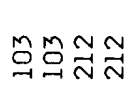 & 읔읔콫 & ลัละ゚ & 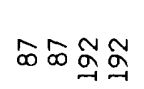 & 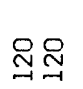 & 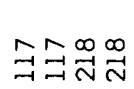 \\
\hline 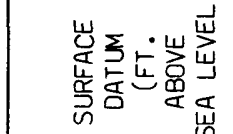 & $\begin{array}{l}8888 \\
\dot{000} 0\end{array}$ & $\begin{array}{l}8888 \\
0000\end{array}$ & $\begin{array}{l}8888 \\
\dot{0} 000\end{array}$ & $\begin{array}{l}8888 \\
\dot{0} 0.00\end{array}$ & $\begin{array}{l}8888 \\
0000\end{array}$ & $\begin{array}{l}8888 \\
\dot{0} 0.00\end{array}$ & $\begin{array}{l}8888 \\
0000\end{array}$ & $\begin{array}{l}8: 8 \\
0 \stackrel{0}{\circ}\end{array}$ & $\begin{array}{l}888.8 \\
9000\end{array}$ \\
\hline 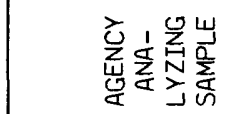 & 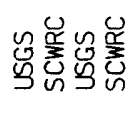 & 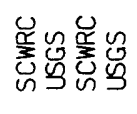 & 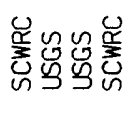 & 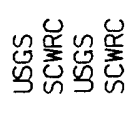 & 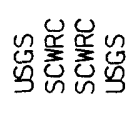 & 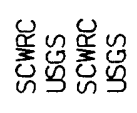 & 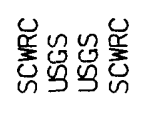 & 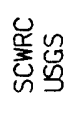 & 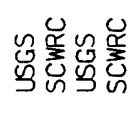 \\
\hline 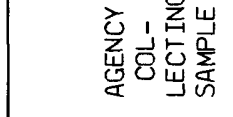 &  & 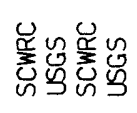 & 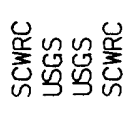 & 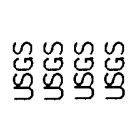 & 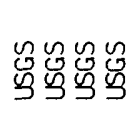 & 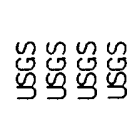 & 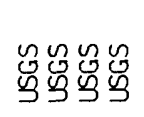 & $\begin{array}{l}\mathscr{y} 心 \\
\dddot{g} \\
\dddot{g}\end{array}$ & 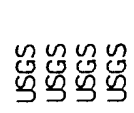 \\
\hline$\sum_{\Sigma}$ & 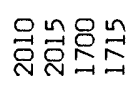 & 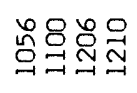 & 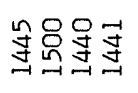 & 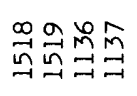 & 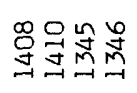 & 品守号号品 & 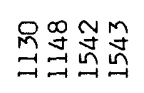 & 总 & 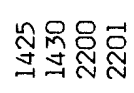 \\
\hline 岕台岕 & 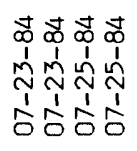 & 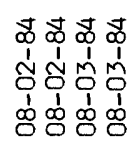 & 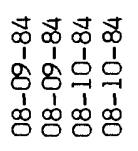 & 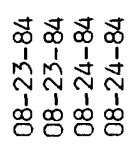 & 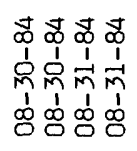 & 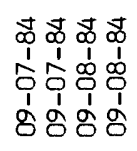 & 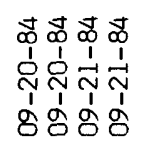 & 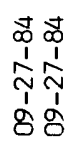 & 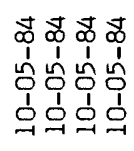 \\
\hline 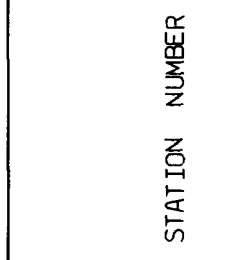 & 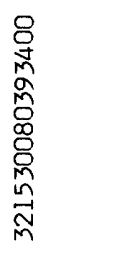 & 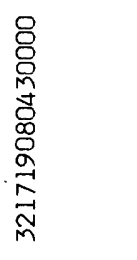 & 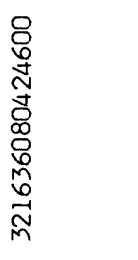 & 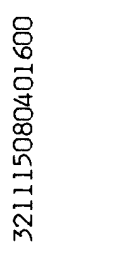 & 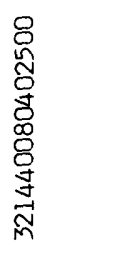 & 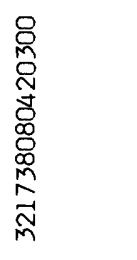 & 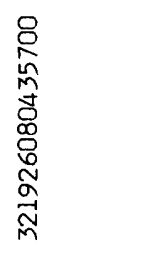 & 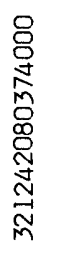 & 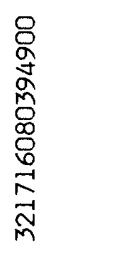 \\
\hline & $\begin{array}{l}\frac{N}{6} \\
\frac{1}{1} \\
\frac{1}{0}\end{array}$ & $\begin{array}{l}m \\
\hat{0} \\
\frac{1}{1} \\
\frac{5}{0}\end{array}$ & $\begin{array}{l}\frac{1}{N} \\
\frac{1}{1} \\
\frac{1}{0} \\
\frac{1}{0}\end{array}$ & $\begin{array}{l}\frac{n}{6} \\
\frac{1}{1} \\
\frac{5}{\infty}\end{array}$ & $\begin{array}{l}0 \\
5 \\
\frac{1}{1} \\
\frac{1}{0}\end{array}$ & $\begin{array}{l}\hat{n} \\
\frac{1}{1} \\
\frac{1}{0} \\
0\end{array}$ & $\begin{array}{l}\infty \\
0 \\
0 \\
-1 \\
\frac{1}{0} \\
0\end{array}$ & $\begin{array}{l}2 \\
6 \\
0 \\
\frac{1}{1} \\
0 \\
0\end{array}$ & $\begin{array}{l}0 \\
0 \\
0 \\
\rightarrow 1 \\
1 \\
\qquad \\
0 \\
0\end{array}$ \\
\hline
\end{tabular}




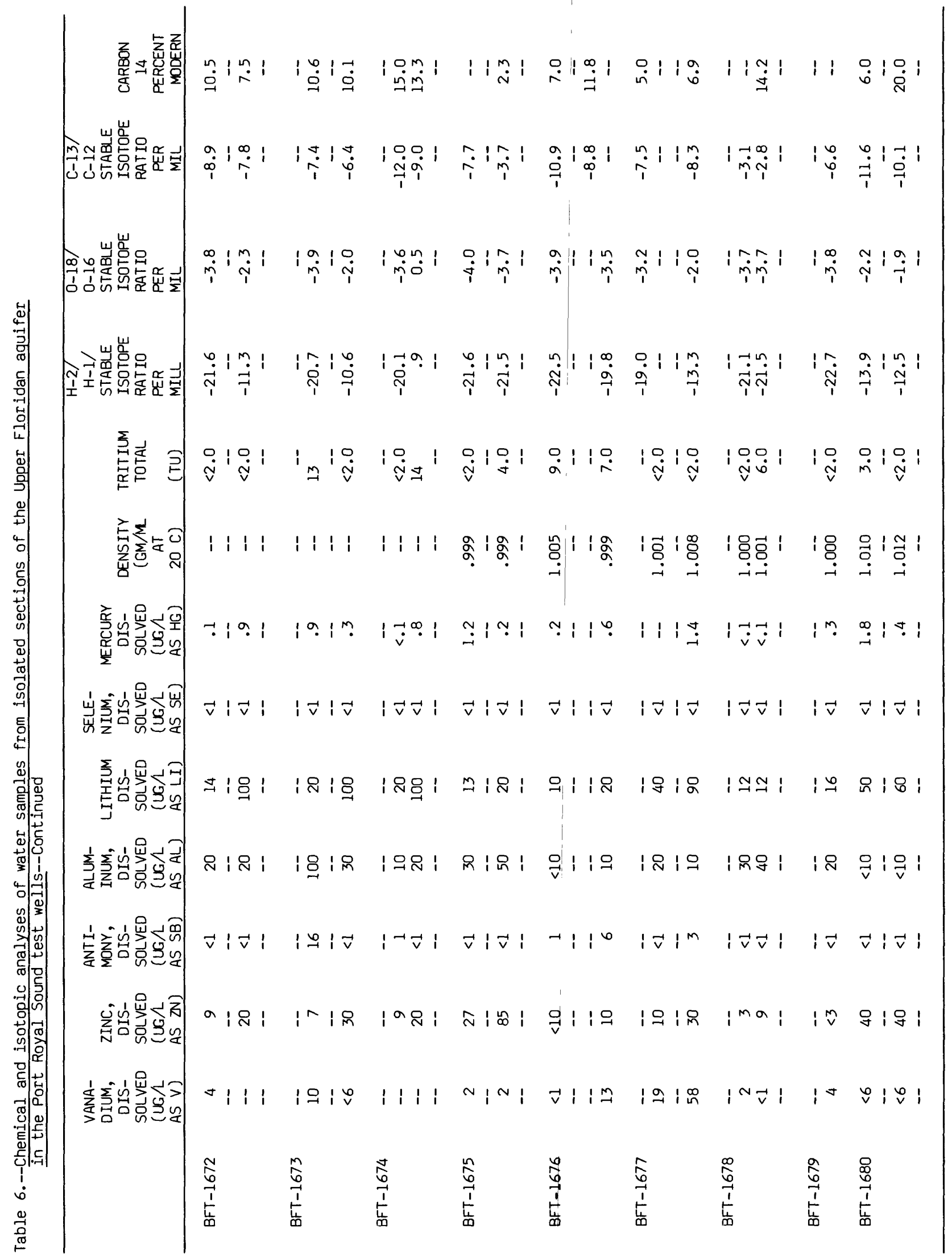




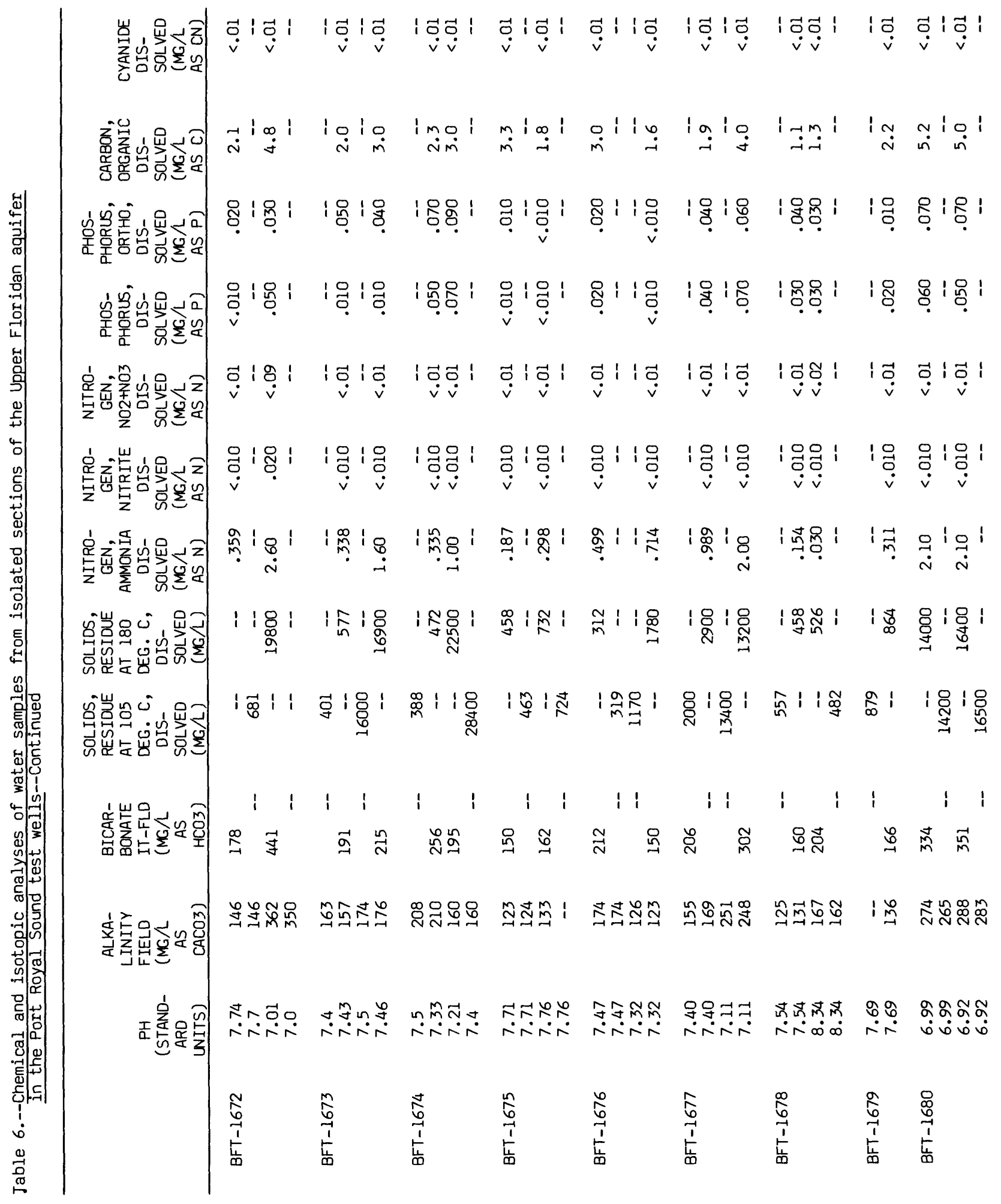




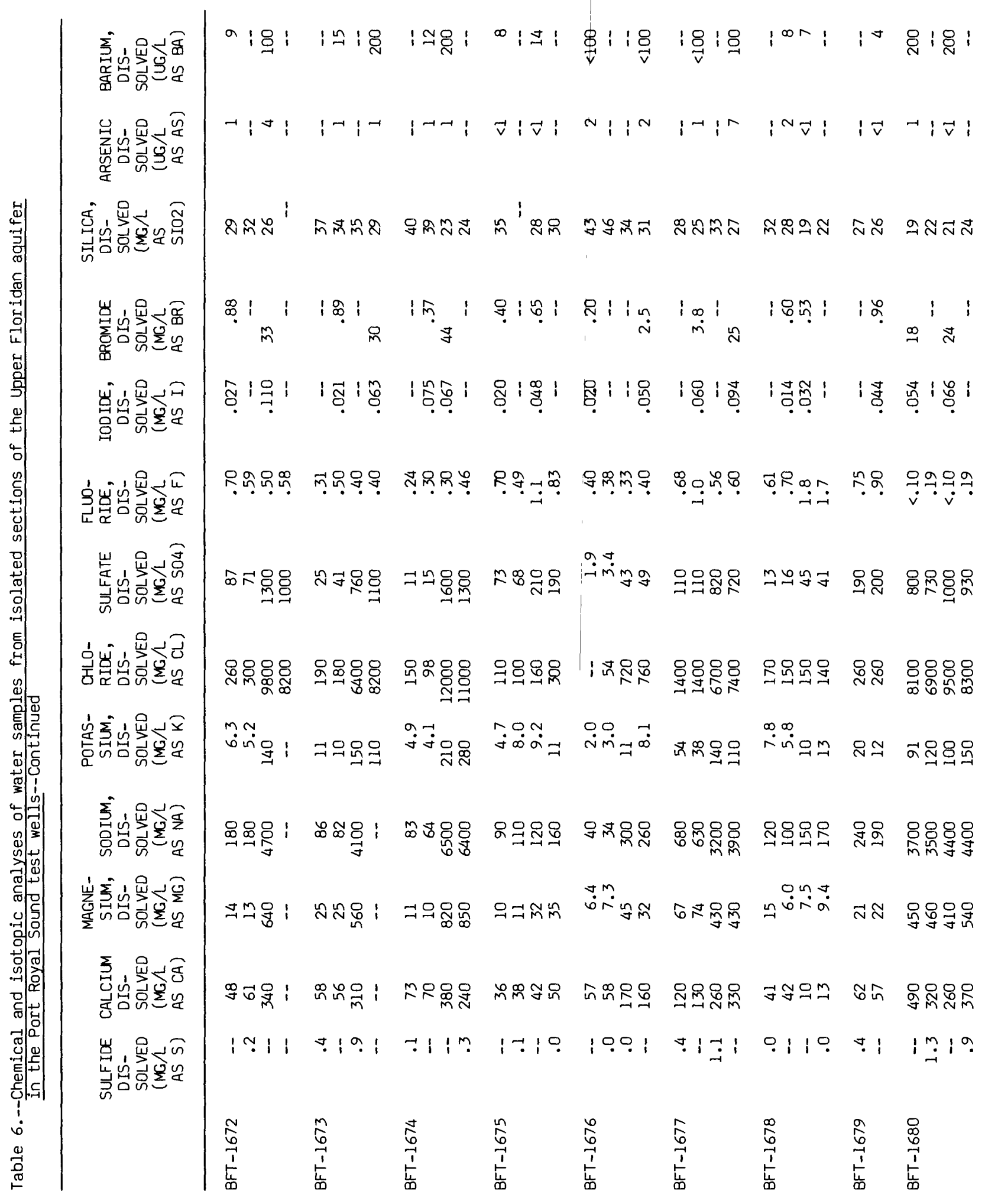




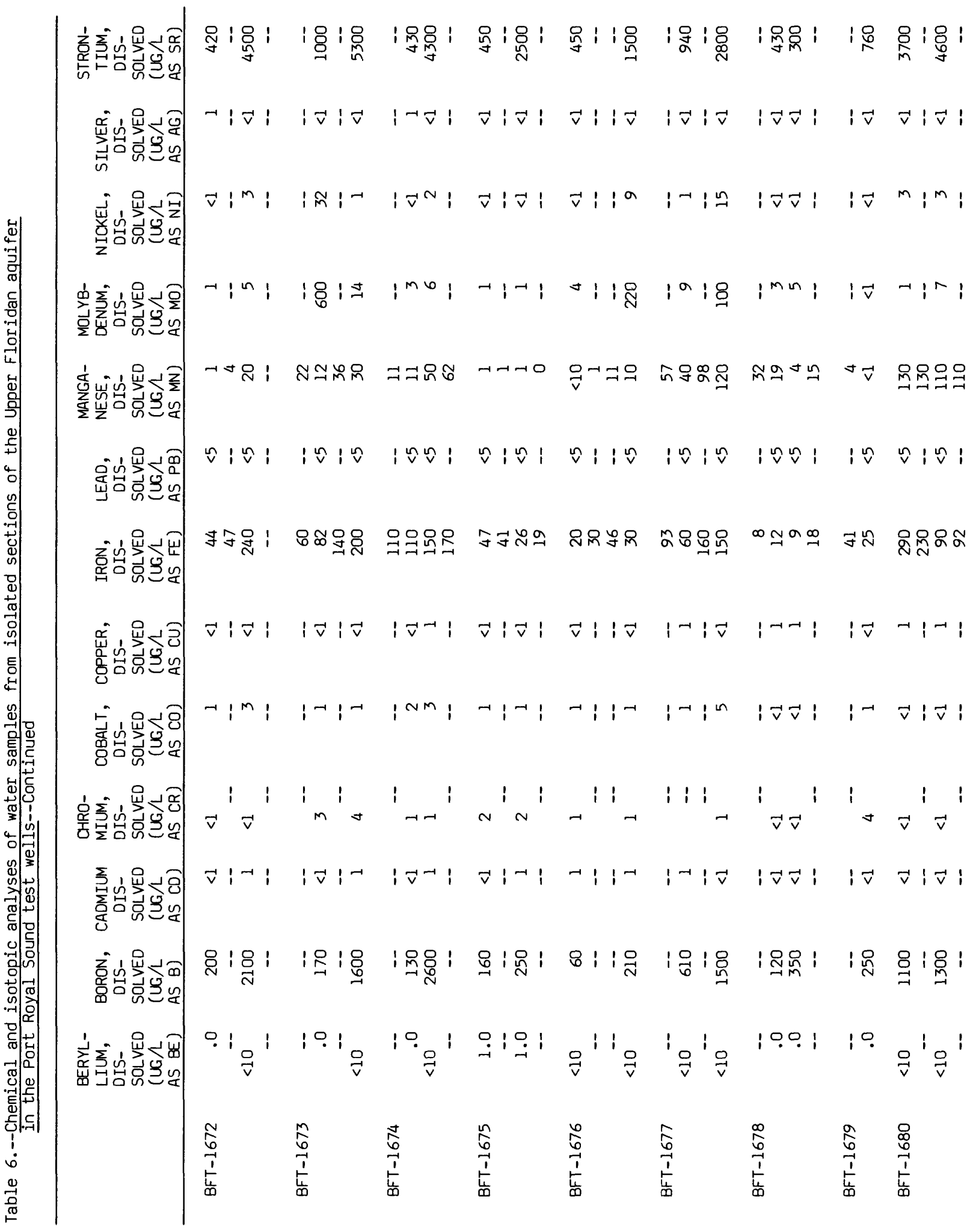


Table 7.--Concentration of chloride in water samples collected from the annulus at the wellhead during drilling

\begin{tabular}{|c|c|c|c|c|}
\hline Well & Date & Time & $\begin{array}{l}\text { Altitude } \\
\text { at bottom } \\
\text { of well } \\
\text { (ft below } \\
\text { sea level) }\end{array}$ & $\begin{array}{c}\text { Chloride } \\
(\mathrm{mg} / \mathrm{h})\end{array}$ \\
\hline \multirow[t]{2}{*}{ BFT-1672 } & $07-23-84$ & $\begin{array}{l}1645 \\
1747\end{array}$ & $\begin{array}{l}106 \\
117\end{array}$ & $\begin{array}{l}310 \\
270\end{array}$ \\
\hline & $07-24-84$ & $\begin{array}{l}1600 \\
1613 \\
1625 \\
1640 \\
1645 \\
1700 \\
1710 \\
1713 \\
1730 \\
1800 \\
1820 \\
1845\end{array}$ & $\begin{array}{l}133 \\
143 \\
153 \\
163 \\
168 \\
173 \\
177 \\
183 \\
193 \\
198 \\
203 \\
211\end{array}$ & $\begin{array}{r}320 \\
600 \\
2,700 \\
3,800 \\
5,500 \\
6,900 \\
8,200 \\
8,800 \\
9,300 \\
9,700 \\
6,400 \\
3,900\end{array}$ \\
\hline BFT -1673 & $08-01-84$ & $\begin{array}{l}1330 \\
1500\end{array}$ & $\begin{array}{r}99 \\
101\end{array}$ & $\begin{array}{l}240 \\
240\end{array}$ \\
\hline BFT -1673 & $08-02-84$ & $\begin{array}{l}1209 \\
1315 \\
1328 \\
1405 \\
1514 \\
1542 \\
1550 \\
1606 \\
1619 \\
1633 \\
1644 \\
1702\end{array}$ & $\begin{array}{l}106 \\
109 \\
116 \\
126 \\
136 \\
141 \\
151 \\
161 \\
171 \\
181 \\
191 \\
201\end{array}$ & $\begin{array}{r}190 \\
160 \\
190 \\
270 \\
240 \\
270 \\
640 \\
1,500 \\
3,700 \\
4,600 \\
4,100 \\
4,900\end{array}$ \\
\hline BFT-1674 & $08-09-84$ & $\begin{array}{l}1631 \\
1643 \\
1700 \\
1730 \\
1905 \\
1918 \\
1940\end{array}$ & $\begin{array}{l}123 \\
133 \\
143 \\
149 \\
159 \\
169 \\
174\end{array}$ & $\begin{array}{r}48 \\
32 \\
40 \\
45 \\
220 \\
5,400 \\
8,600\end{array}$ \\
\hline
\end{tabular}


Table 7.--Concentration of chloride in water samples collected from the annulus at the wellhead during drilling--Continued

\begin{tabular}{|c|c|c|c|c|}
\hline Well & Date & Time & $\begin{array}{l}\text { Altitude } \\
\text { at bottom } \\
\text { of well } \\
\text { (ft below } \\
\text { sea level) }\end{array}$ & $\begin{array}{l}\text { Chloride } \\
(\mathrm{mg} / \mathrm{L})\end{array}$ \\
\hline BFT-1675 & $08-23-84$ & $\begin{array}{l}1315 \\
1623 \\
1631 \\
1637 \\
1638 \\
1639 \\
1708 \\
1712 \\
1725 \\
1734 \\
1745 \\
1813\end{array}$ & $\begin{array}{r}97 \\
113 \\
123 \\
133 \\
143 \\
153 \\
163 \\
173 \\
183 \\
193 \\
203 \\
213\end{array}$ & $\begin{array}{l}270 \\
110 \\
110 \\
130 \\
110 \\
110 \\
120 \\
130 \\
130 \\
130 \\
140 \\
290\end{array}$ \\
\hline BFT-1676 & $08-30-84$ & $\begin{array}{l}1510 \\
1520 \\
1525 \\
1545 \\
1550 \\
1605 \\
1615 \\
1630 \\
1700 \\
1907\end{array}$ & $\begin{array}{l}120 \\
130 \\
140 \\
150 \\
160 \\
170 \\
180 \\
190 \\
200 \\
210\end{array}$ & $\begin{array}{r}50 \\
52 \\
65 \\
58 \\
64 \\
67 \\
84 \\
170 \\
550 \\
830\end{array}$ \\
\hline BFT-1677 & $09-07-84$ & $\begin{array}{l}1438 \\
1445 \\
1455 \\
1522 \\
1536 \\
1550 \\
1605\end{array}$ & $\begin{array}{l}109 \\
119 \\
129 \\
139 \\
149 \\
159 \\
169\end{array}$ & $\begin{array}{l}1,500 \\
1,700 \\
2,100 \\
3,000 \\
4,100 \\
5,400 \\
6,400\end{array}$ \\
\hline
\end{tabular}


Table 7.--Concentration of chloride in water samples collected from the annulus at the wellhead during drilling--Continued

\begin{tabular}{|c|c|c|c|c|}
\hline Well & Date & Time & $\begin{array}{l}\text { Altitude } \\
\text { at bottom } \\
\text { of well } \\
\text { (ft below } \\
\text { sea level) }\end{array}$ & $\begin{array}{c}\text { Chloride } \\
(\mathrm{mg} / \mathrm{L})\end{array}$ \\
\hline $\mathrm{BFT}-1678$ & $09-20-84$ & $\begin{array}{l}1304 \\
1324 \\
1350 \\
1414 \\
1455 \\
1604 \\
1720 \\
1757 \\
1808 \\
1834 \\
1846 \\
1906 \\
1920\end{array}$ & $\begin{array}{r}92 \\
97 \\
102 \\
112 \\
118 \\
127 \\
137 \\
142 \\
152 \\
162 \\
172 \\
182 \\
192\end{array}$ & $\begin{array}{r}240 \\
240 \\
160 \\
160 \\
150 \\
150 \\
160 \\
160 \\
150 \\
140 \\
140 \\
100 \\
87\end{array}$ \\
\hline BFT-1679 & $09-27-84$ & $\begin{array}{l}1549 \\
1556 \\
1610 \\
1617 \\
1624 \\
1630 \\
1654 \\
1707 \\
1730\end{array}$ & $\begin{array}{l}130 \\
140 \\
150 \\
160 \\
170 \\
180 \\
190 \\
200 \\
210\end{array}$ & $\begin{array}{l}260 \\
250 \\
230 \\
230 \\
220 \\
240 \\
240 \\
260 \\
270\end{array}$ \\
\hline$B F T-1680$ & $10-05-84$ & $\begin{array}{l}1500 \\
1515 \\
1545 \\
1600 \\
1615 \\
1630 \\
1640 \\
1652 \\
1700 \\
1800\end{array}$ & $\begin{array}{l}128 \\
138 \\
148 \\
158 \\
168 \\
178 \\
188 \\
198 \\
208 \\
218\end{array}$ & $\begin{array}{l}7,000 \\
7,200 \\
7,500 \\
7,400 \\
8,000 \\
8,100 \\
8,000 \\
8,100 \\
8,100 \\
8,000\end{array}$ \\
\hline
\end{tabular}

